\title{
A modeling strategy for integrated batch process development based on mixed-logic dynamic optimization
}

\author{
Marta Moreno-Benito ${ }^{\mathrm{a}, *}$, Kathrin Frankl ${ }^{\mathrm{b}}$, Antonio Espuña ${ }^{\mathrm{c}}$, Wolfgang Marquardt ${ }^{\mathrm{b}}$ \\ ${ }^{a}$ Centre for Process Systems Engineering, University College London, Torrington Place, London WC1E $7 J E$, UK \\ ${ }^{b}$ Process Systems Engineering, RWTH Aachen, Turmstraße 46, D-52056 Aachen, Germany \\ ${ }^{c}$ Department of Chemical Engineering, Universitat Politècnica de Catalunya, ETSEIB, Av. Diagonal 647, E-08028 \\ Barcelona, Spain
}

\begin{abstract}
This paper introduces an optimization-based approach for the simultaneous solution of batch process synthesis and plant allocation, with decisions like the selection of chemicals, process stages, task-unit assignments, operating modes, and optimal control profiles, among others. The modeling strategy is based on the representation of structural alternatives in a State-Equipment Network (SEN) and its formulation as a mixed-logic dynamic optimization (MLDO) problem. Particularly, the disjunctive multistage modeling strategy by Oldenburg and Marquardt [1] is extended to combine and organize single-stage and multistage models for representing the sequence of continuous and batch units in each structural alternative and for synchronizing dynamic profiles in input and output operations for material transference. Two numerical examples illustrate the application of the proposed methodology, showing the enhancement of the adaptability potential of batch plants and the improvement of global process performance thanks to the quantification of interactions between process synthesis and plant allocation decisions.
\end{abstract}

Keywords: batch process synthesis, plant allocation, dynamic optimization, generalized disjunctive programming, synchronization, multistage modeling

\section{Introduction}

Specialty chemical industries are meant to assimilate and execute many different processes along their lifecycle, as many specialty products are subject to quickly changing markets or may be displaced by rapidly advancing technologies. The fast introduction of new products and processes into production facilities is crucial to be first in the marketplace, even if such processes should be sustainable in order to ensure a long-term economic viability and be competitive in front of stockholders. The investment of time and resources to design, construct, and validate a new plant each time that a different product is approved for

\footnotetext{
${ }^{*}$ Corresponding author: Tel.: +442076793836, Fax: +442076797092

Email address: marta.moreno.benito@ucl.ac.uk (Marta Moreno-Benito)
} 
commercialization is not a viable strategy in most cases. This way, batch systems are highly appropriate for specialty chemical sector because their inherent flexibility allows their adaptation and reconfiguration to incorporate new production lines with none or only partial plant modifications.

The integrated batch process development problem involves the synthesis of processing schemes and the allocation of manufacturing facilities, once a new product has been discovered and its production opportunity has been recognized $[2,3]$. Both sub-problems have a number of degrees of freedom closely interrelated, which ultimately define the process and the required equipment investment in new or existing manufacturing facilities. For instance, the development of a batch process stage could include decisions like the operating mode for determining the process structure -i.e. parallel, series or single unit operation- in order to exploit the adaptability potential of the batch system. This decision is closely related to the task-unit assignment and equipment selection, especially in the case of existing plants with a given set of units and connecting pipelines. Simultaneously, each equipment unit has to accommodate the assigned tasks using a batch or semi-continuous procedure, which involves either a chain of batch operations or an intermittent use of continuously operated plant elements, respectively. This way, batch operations are defined by their duration and dynamic feed-forward trajectories of control variables, and their feasibility depends on physical constraints like the capacity of the selected units. Additionally, it is necessary to determine the batch size and the number of batches, as they are responsible of the total demand satisfaction and are interrelated with the equipment capacity and the process conversion. Other degrees of freedom for full process development include the selection of process stages, the recirculation of intermediate flows, the installation of buffer tanks for working with different cycle times or batch sizes in successive process stages, the selection among alternative technologies, and the selection of chemicals.

Given the above complexity, the problem of batch process development is generally solved through decomposition strategies or using fixed processing recipes and approximated models. This way, some academic studies address the synthesis of batch processes by disregarding the subsequent allocation of equipment items $[4,5,6,7,8,9]$, whilst some contributions include allocation decisions by means of sequential decision-making procedures $[10,11,12,13]$. Other works focus on the optimal operation of individual units through the application of tools like dynamic optimization (DO) $[14,15]$ with different kinds of decision variables, namely the dynamic profiles of control variables, the durations of batch operations [16], and the structural decisions that determine the configuration of processing units $[17,18]$. The design of batch plants is another area of extensive research $[19,20]$ where the allocation of manufacturing facilities and plant sizing are usually solved using fixed recipes or approximated models for simplifying the process stage representation [21].

The aforementioned partial solutions of the batch process development problem are due to the high mathematical and computational complexity for the modeling and solution of that problem. The batch nature of the process involves not only the combinatorial assessment for linking equipment and task networks [22], but also the adjustment of processing conditions along time in each process stage, requiring dynamic 
models for process performance representation as well as dynamic profiles of control variables to fully explore the attainable region [14]. Process operations also require the representation of discrete events that determine the transition of batch operations [23]. Moreover, qualitative information has to be included regarding decisions like task selection, sequencing, splitting, equipment assignment, or chemicals selection. Finally, batch integrity has to be guaranteed for every structural processing alternative by synchronizing the material transference between batch and semi-continuous plant elements.

However, divide and conquer strategies jeopardize the efficiency of the resulting process. On the one hand, the sequential solution of batch process development involves losing a significant part of the interaction among the degrees of freedom, thus restricting the system trade-off evaluation according to global industrialscale objectives [24]. On the other hand, the direct implementation of fixed recipes in existing equipment may force its operation in extreme and even infeasible conditions [25]. Overall, in order to fully optimize the processes and guarantee the best utilization of available equipment and new investments, process synthesis and plant allocation should be solved simultaneously.

This paper presents a novel modeling strategy to optimize the integrated batch process development problem by means of a comprehensive mixed-logic dynamic optimization (MLDO) model that evaluates the interactions between synthesis and allocation decisions simultaneously. For instance, other studies have addressed this problem by means of DO and MIDO formulations to optimize the reference trajectories of control variables [26, 27], although they involve the pre-specification of the sequence of process stages and allocation decisions. Other approaches simplify the problem through the approximation of the batch process behavior using algebraic models - e.g. screening models [28] and posynomial functions [29]. In some cases, these approaches have been complemented with the optimization of the dynamic feed-forward trajectories of control variables, albeit relying on the iterative evaluation of structural and performance decisions rather than on the simultaneous solution [30]. In contrast, other works opt for constant set-points for defining the processing conditions [31, 32]. Additionally, several authors have sought the sustainable synthesis of processing schemes in retrofit scenarios, even if they use heuristic approaches based on path flow decomposition and indicator-based assessment [33, 34, 35, 36, 37]. Besides, the use of dynamic performance models in allocation problems has received a strong impulse in the last two decades in the context of shortterm scheduling, even though these contributions do not cover other synthesis and design decisions, such as the selection of process technologies, chemicals, or equipment sizing, among others [38, 39, 40, 41, 42]. Regarding the synchronization of consecutive process stages, it is only in the context of scheduling and plant design that this feature is explicitly addressed, although the transference time interval and the dynamic transference profiles required in input and output operations are approximated as a single time point [43, 44].

Our goal in this study is the systematic optimization of batch processes according to global targets in changing frameworks, by taking the maximum advantage of new and previously existing equipment in grassroots and retrofit scenarios. The integrated batch process development problem here studied (§ 2) 
comprises process synthesis decisions like the selection of process stages or tasks, their splitting into subtasks, the technological specification, the selection of chemicals, the definition of feed-forward control trajectories and duration of batch operations, the definition of intermediate flow recycles, and the synchronization of material transfer operations. Simultaneously, the problem includes plant allocation decisions, namely the equipment selection, the task-unit assignment, the definition of the operating mode in single, series, or parallel operation, and the batch sizing. Finally, equipment capacities may represent either model constraints in existing processing items or additional decisions in equipment investments.

Essentially, the modeling strategy $(\S 3)$ relies on the representation of processing alternatives in a StateEquipment Network (SEN) superstructure [45] and its mathematical formulation as a MLDO problem (§ 4). In particular, the combination of hybrid discrete/continuous modeling and generalized disjunctive programming proposed by Oldenburg and Marquardt [1] is here extended to incorporate: (i) the combination of single-stage and multistage models to represent continuous and batch processing elements, (ii) the definition of multiple representation levels to link the timing among the different batch unit procedures, whose ordering is not know beforehand, and (iii) the synchronization of flow rates and compositions in material transfer operations as a function of the selected process structure and tasks performance. To the authors' knowledge, the combination of these modeling elements to handle all the aforementioned decisions simultaneously has not hitherto been applied in the context of batch process design. Next, the resulting MLDO model can be solved through several solution methods, such as the direct-simultaneous approach (§5). The benefits associated to the proposed optimization-based strategy for integrated batch process development are illustrated with two numerical examples ( $\S 6$ and $\S 7$ ).

\section{Problem statement}

This work solves simultaneously the batch process synthesis and the plant allocation sub-problems in single-product campaigns, in order to define the process and the required equipment investments in new or existing manufacturing facilities, while enhancing the process efficiency and plant flexibility compared to the use of fixed recipes. The entire problem is defined as follows.

Given:

- Planning data: set of final products, intermediates, and raw materials, expected demand of final products, and maximum time horizon;

- Plant diagram: the SEN superstructure of available and potentially installed equipment units for each process stage, pipelines and connection nodes like mixers and splitters;

- Task network: potential and mandatory process stages, alternative chemicals involved in each process stage -i.e. reactants, solvents, or catalysts-, allowed technologies, and possible reuse of intermediates in following batches; 
- Batch process operation: allowed task-unit assignments, batch operations within each unit procedure, operation to operation switching conditions, and set of limiting processing conditions for each unit;

- Process dynamics: DAE systems to represent the process behavior in each unit procedure, initial conditions, and set of process variables and dynamic or time-invariant controls;

- Data related to performance evaluation: decision criteria and specific data to evaluate the objective function $-e . g$. selling price of final product, direct cost of raw materials, investment, amortization, operating costs in processing units, environmental impact indicators;

the goal is to determine:

- Synthesis of processing schemes decisions: selection of process stages and splitting into subtasks, technological specification, chemicals involved -i.e. reactants, solvents, or catalysts-, reference trajectories of the feed-forward control variables, duration of batch operations composing each process stage, recirculation of intermediate flows to be used in the next batch, and material transfer synchronization between tasks -i.e. synchronization of flow rates, compositions, and starting and final times;

- Allocation of manufacturing facilities decisions: selection of processing and storage units, taskunit assignment, operating mode -i.e. single, series, or parallel operation-, and batch sizes;

- Plant design decisions: equipment sizing;

such that the adopted performance metrics are optimized. This problem can be solved in grassroots and retrofit scenarios. In the second case, the already existing equipment units have a predefined size value unless their capacity expansion is considered. For the sake of completeness, it is worth noting that there are other process development decisions which are out of the scope of this study, as it is the case of decisions associated to multiproduct and multipurpose campaigns, e.g. batch order.

\section{Modeling strategy}

The solution of optimization problems with structural decisions is usually addressed through the representation of all processing alternatives in a superstructure, which is later formulated into a mathematical model, to be finally optimized [46]. In this work, a novel modeling strategy is used for representing all the degrees of freedom and interactions that characterize the integrated batch process development problem in a holistic optimization model. The strategy is based on a bi-level SEN representation and its formulation as a MLDO problem. The background of mathematical tools for understanding such strategy is following presented. 


\subsection{Background}

Superstructure representation. Batch design and scheduling problems require superstructure representations that combine plant, process, and material states. The most popular proposals are the State-Task Network (STN) [47], the Resource-Task Network (RTN) [48], and the State-Equipment Network (SEN) [45]. Among them, the SEN entails the representation of equipment items and transfer material states, enabling the formulation of equipment equations and showing the dependence of material transference profiles on processing paths and conversion of preceding process stages. Precisely, other contributions in the literature have applied SEN superstructures to solve scheduling problems with process dynamics [39, 49].

Hybrid discrete/continuous models. These are dynamic models that represent batch process performance and batch events by combining discrete and continuous variables [50]. Depending on the type of discontinuities represented, they are classified as: (i) single-stage models, without discrete events, (ii) multistage models, with explicit discontinuities, and (iii) general hybrid discrete/continuous models, with implicit and explicit discontinuities. Hybrid discrete/continuous models have been widely used in DO problems for determining the optimal control profiles of individual processing units along time $[14,15]$. The optimization of dynamic control trajectories in given chains of process stages has been also applied in the context of batch process synthesis [26, 30, 27], batch plant design [38, 51], and short-term scheduling [39, 40, 41, 42], among others.

Logic-based modeling. The incorporation of logical variables and equations into mathematical models allows the combination of quantitative and qualitative information. The use of logics in optimization problems was originally proposed by Balas [52] in the so-called disjunctive programming, and rapidly became an alternative to mixed-integer modeling for representing structural decisions [53, 54]. This kind of problems were later formalized as generalized disjunctive programming (GDP) $[55,56]$, where qualitative decisions are mathematically represented using Booleans and available process knowledge and heuristic rules are formulated through logical propositions. Mixed-logic formulations involving dynamic equations have been also applied to several problems, such as model predictive control [57], simultaneous design and control [58], and scheduling of continuous processes with grade transitions [59]. In the context of batch process development, Linninger and Chakraborty [31,32] addressed the problem of batch process synthesis and plant allocation including logical rules in the superstructure formulation, but simplifying the problem through approximated process performance models. In contrast, Oldenburg et al. $[1,17]$ presented a MLDO formulation that combined hybrid discrete/continuous models and GDP for solving the optimal control profiles, configuration, sequencing, and operation of batch equipment units with structural decisions. However, the formulation only considered individual units rather than task sequences, so the ordering and synchronization of processing units was not approached. 
Coexisting single-stage and multistage models for synchronization purposes. Single-stage and multistage models can be used to represent batch and semi-continuous procedures in the superstructure, respectively. Since it is necessary to guarantee batch integrity, this kind of models have to be linked to each other in material transfer operations and synchronized as a function of the selected sequence of processing units. However, currently there are neither modeling frameworks nor software tools which can handle coexisting multistage and single-stage models. Previous works on optimization of processing unit sequences with dynamic profiles could avoid the ordering and combination of several multistage models associated to batch units either because the physical constraints of existing equipment units were not considered [26] or because batch process stages were represented by a single batch operation and the whole process could be represented by a unique multistage model -i.e. by associating one modeling stage with each task [60].

\subsection{Bi-level SEN representation and MLDO modeling strategy}

In this context, the proposed modeling strategy is based on the representation of processing alternatives in a SEN superstructure, which is divided into two representation levels and formulated mathematically as a MLDO problem. The SEN superstructure represents the interconnection of existing and potential units and transition states according to the plant diagram, e.g. Fig. 1. This way, the SEN allows the explicit representation of physical plant restrictions and procedures of processing units as well as the definition of material transfer states as a function of selected units, their order, and their conversion.

[Figure 1 about here.]

The mathematical formulation of the superstructure and all the associated decisions into a MLDO problem is addressed by defining the problem disjunctions, logical propositions, and nested hybrid discrete/continuous models -referred to as multistage models in this paper-, as it was proposed by Oldenburg and Marquardt [1] for the optimization of individual units. In this contribution, the MLDO formulation is extended to combine, organize, and synchronize the multiple equipment units in the SEN superstructure according to the processing alternative that is selected. With this purpose, each unit is represented with either a single-stage or a multistage model associated to a semi-continuous or batch operation respectively. In this work, each mathematical stage corresponds to a batch operation. Moreover, in order to perform the coordination and timing of consecutive units, these are distributed into two representation levels, which are optimized simultaneously, dividing the superstructure into Level 1, containing batch units, and Level 0, with connection nodes, storage tanks, and semi-continuous processing units, as shown in Fig. 2. This modular bi-level representation allows the ordering of processing elements as a function of decisions like the taskunit assignment because, this way, the time coordinates of batch units within the integrated optimization problem can be moved with respect to the time horizon of other batch units and the complete process. The novel coexistence and synchronization of single-stage and multistage models is illustrated in Fig. 3a. The 
synchronization in terms of time is defined through disjunctive equations and logical propositions in the formulation.

[Figure 2 about here.]

[Figure 3 about here.]

Additionally, a specific treatment of the equations that compose the optimization problem is performed in order that standard optimization tools can handle the relations among the mathematical stages that represent batch operations:

1. Transformation of single-stage models at Level 0 into multistage ones. The connection across unit procedures models is first established by transforming single-stage models at Level 0 into a multistage model that includes all potential batch operations at Level 1, as represented in Fig. 3b. To do so, the set of equations of the original single-stage model is replicated for each new stage and the continuity of process variables is guaranteed by incorporating stage-to-stage relations.

2. Normalization of mathematical stages. Since the several processing elements at Levels 0 and 1 may be composed of a different number of mathematical stages with different duration (see Fig. 3b), they should be homogenized by normalizing their time axis. This way, it is possible to handle the several stage sequences by means of one normalized time axis, as shown in Fig. 3c. The normalization of time intervals is carried out by multiplying the differential equations by the stage duration and redefining their derivation interval from 0 to 1 . Except for the actual first stage, the initial conditions of all other stages should be treated as degrees of freedom whose values are subject to the fulfillment of stage-to-stage continuity equations.

3. Explicit definition of stage durations. Finally, it is necessary to express stage durations as explicit variables in order that the time equivalence between synchronized stages can be established across processing elements. This is accomplished with the normalization of time intervals, as defined in the previous step.

Beyond the application of these transformations to the multistage model equations, the identity of each stage at Levels 0 and 1 is preserved, since it is a key issue for the synchronization of unit procedures.

\section{Problem formulation}

The variables and equations that compose the optimization model for integrated batch process development are following detailed, according to the aforementioned modeling strategy $(\S 3)$. 


\subsection{Notation}

The required sets, parameters, and control variables to interrelate all the problem elements in the formulation are following defined. Batch units $j \in U$ are synchronized as a function of their processing order by relating their mathematical stages $k \in K_{j}$ at Level 1 to general stages $l \in L$ at Level 0 in synchronization equations. The number of stages that are necessary at Level 0 is subject to the selected process stages $i \in P S$ and their operating modes $\psi \in \Psi_{i}$. For instance, operation in a single batch unit $U_{1}$ in the superstructure of Fig. 1 can involve the three operations load, hold, and unload unit $U_{1}$, as represented in Fig. $4 \mathrm{a}$ (configuration $\alpha$ ). In contrast, two additional stages are likely to be required in series operation to complete the hold and unload operations of a second batch unit $U_{2}$, as illustrated in Fig. $4 \mathrm{~d}$ (configuration $\sigma$ ). The maximum number of stages $L^{\max }$ concerning all structural alternatives corresponds to the solution with all batch units in series. The total time $T^{f}$ at Level 0 is then divided into $L^{\max }$ intervals with different durations, as shown in Fig. 4 for this example. Out of the resulting set of stages $L=\left\{1, \ldots, L^{\text {max }}\right\}$, only the subset $L^{a}$ composed by the so-called active stages is effective in each structural alternative.

[Figure 4 about here.]

The connection between Levels 0 and 1 is especially relevant on stages associated to operations of batch units $j \in U$ with material transference. These stages are termed input and output stages and are denoted by $I_{j} \subseteq K_{j}$ and $O_{j} \subseteq K_{j}$ respectively. Additionally, input and output pipelines of batch units are represented at both Level 0 (represented by $n \in N_{j}^{\text {in }} \cup N_{j}^{\text {out }}$ ) and Level 1 (represented by $m \in M_{j}^{\text {in }} \cup M_{j}^{\text {out }}$ ) and are related across the two levels in the formulation. Essentially, the characterization of input and output pipelines is associated to flow rates $F_{m, k}^{j}(t)$ and compositions $x_{c, m, k}^{j}(t)$, which are referred to as input and output variables. As it is illustrated in Fig. 5, the input and output flow rates should be restricted to zero in those stages that are not input $K_{j} \backslash I_{j}$ or output $K_{j} \backslash O_{j}$ stages respectively, in order to guarantee the absence of material transfer. The system state inside a unit depends uniquely on its input and output variables, internal control variables, and dynamic model.

[Figure 5 about here.]

Control variables. According to their mathematical nature, decision variables are classified into four categories:

1. Dynamic control variables $u_{k}^{d y n}(t)$ : flow rates $F_{m, k}^{j}(t)$ in input and output pipelines $m \in M_{j}^{\text {in }} \cup M_{j}^{\text {out }}$ and internal control variables $i n t_{k}^{j}(t)$ along batch operations $k \in K_{j}$ of batch units $j \in U$;

2. Static or time-invariant decision variables $u^{\text {stat }}$ : duration $t_{l}$ of batch operations represented by mathematical stages $l$ at Level 0 ;

3. Integer decision variables $u^{\text {int }}$ : number $N B_{p}$ of batches for manufacturing product $p \in P$ and discrete sizing $S i z e^{j}$ of equipment units $j \in U$; 
4. Booleans $u^{B o o l}$ : selection of tasks $Z_{i}$, processing and storage units $Y_{j}$, operating modes $X_{\psi}^{i}$, processing orders $W_{j, q}$, technological specifications $V_{\lambda}^{j}$, chemical compounds $S_{c}^{j}$, and recirculation of intermediate flows $R_{n}$.

The consideration of all these decisions may lead to over-specified problems. Then, in order to improve the efficiency of the search procedure and reduce convergence issues, it is advised to reduce the number of degrees of freedom (DOF) in the optimization problem, by assessing the predefined decisions and equations that relate control variables. Principally, the reduction of DOF can be applied to logical decisions and to dynamic control variables. Specifically, the synchronization of dynamic profiles in operations with material transference is subject to the selected process structure. Therefore, the configuration $\psi \in \Psi_{i}$ of each process stage $i \in P S$ may involve different equations relating flow rates and, as a result, a different number of DOF regarding dynamic profiles (represented in parameter $D O F_{i, \psi}$ ) and dependent batch units (represented in the set $\left.D_{i, \psi} \subseteq U_{i} \subseteq U\right)$. The reduction of the number of control variables is analyzed at the end of this section on the basis of the optimization model.

\subsection{Batch procedures at Level 1}

Models of batch units. Let us consider batch units $j \in U$ located at Level 1 of the superstructure. Their operation and process performance are represented by a $\left|K_{j}\right|$-stage model. For each unit, a two-term disjunction controlled by Boolean $Y_{j}$ is introduced. If $Y_{j}$ is true, this unit is selected to allocate one task and its corresponding $\left|K_{j}\right|$-stage model is activated inside the disjunctive term. In contrast, if $Y_{j}$ is false, the unit is not selected and a bypass strategy [1] is applied, which defines $\left|K_{j}\right|$ equivalent stages where no process takes place. These are termed bypass stages and their purpose is to maintain the same number of mathematical stages in the model, as represented in the Petri net of Fig. 6. To do so, stage durations

$t_{k}^{j}$ are set to zero and dynamic equations and constraints are replaced by a set of equations that cancel the process.

[Figure 6 about here.]

Overall, the disjunctive equation for batch unit $j \in U$ is defined by the following form, bearing in mind 
that the time interval has been normalized (see Fig. 3c):

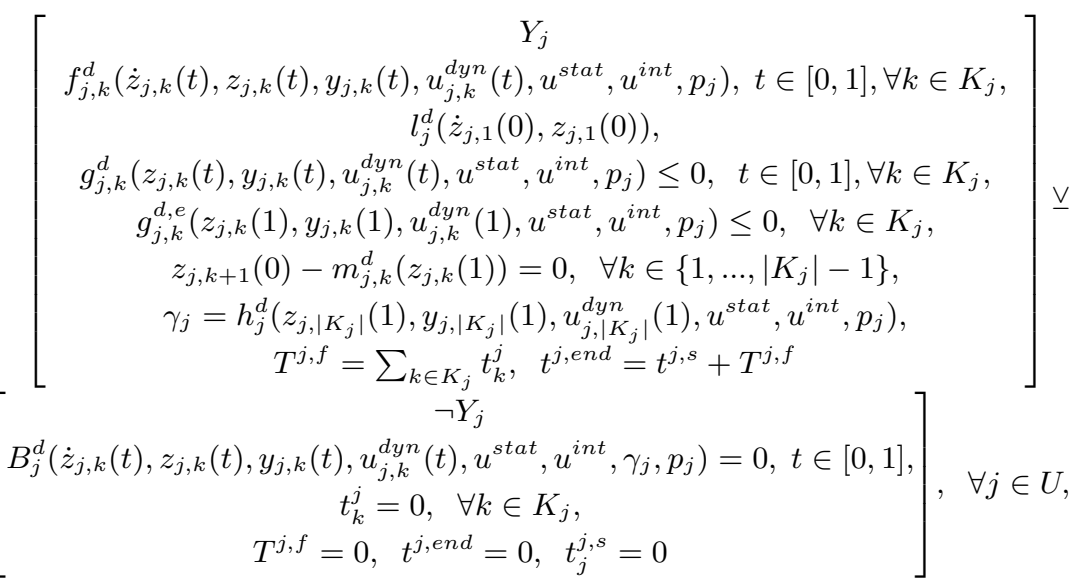

where $f_{j, k}^{d}, g_{j, k}^{d}, g_{j, k}^{d, e}$, and $m_{j, k}^{d}$ are the DAE system, path constraints (PC), end-point constraints (EPC), and stage-to-stage continuity in stage $k \in K_{j}$ of unit $j \in U . l_{j}^{d}$ are the relations that define initial conditions, $h_{j}^{d}$ is the set of equations to calculate time-invariant variables $\gamma_{j}$ evaluated at the final time $(t=1)$ of last stage $\left|K_{j}\right|$, and $B_{j}^{d}$ are the equations that define the system in bypass stages. Each stage $k \in K_{j}$ contains time dependent differential $z_{j, k}(t)$, algebraic $y_{j, k}(t)$, and control $u_{j, k}^{d y n}(t)$ variables, including input/output flow rates $F_{m, k}^{j}(t)$ and compositions $x_{c, m, k}^{j}(t)$ and internal control variables $i n t_{k}^{j}(t)$. The multistage model is also characterized by process parameters $p_{j}$ and time-invariant variables $\gamma_{j}$, which contribute to the evaluation of the objective function and other key performance indicators (KPIs), like product selectivity or conversion in $j \in U$.

To complete batch unit models at Level 1, input/output variables should be dismissed in those stages which are not input/output stages respectively, as illustrated in Fig. 5, by restricting their value to zero. With this purpose, the following equation is defined:

$$
\left[\begin{array}{c}
Y_{j} \\
F_{m^{\text {in }}, k}^{j}(t)=0, \quad \forall m^{\text {in }} \in M_{j}^{\text {in }}, \forall k \in K_{j} \backslash I_{j}, \\
F_{m^{\text {out }}, k}(t)=0, \quad \forall m^{\text {out }} \in M_{j}^{\text {out }}, \forall k \in K_{j} \backslash O_{j}
\end{array}\right], \forall j \in U .
$$

Additionally, the volume $v_{k}^{j}(t)$ of material processed at unit $j \in U$ can not surpass the equipment capacity $S i z e^{j}$ in any stage $k \in K_{j}$, either $S i z e^{j}$ is a free decision variable associated to a newly installed unit or it is a constraint associated to an existing item. This limitation is formulated as:

$$
v_{k}^{j}(t) \leq \operatorname{Size}^{j}, \quad t \in[0,1], \forall k \in K_{j}, \forall j \in U \text {. }
$$

Technological specification. The technological specification is used to distinguish between processing alternatives that can be used for the same unitary operation from the set $\Lambda$. The equipment arrangement that characterize each technology has particular features and then requires specific physicochemical equations, 
parameters, and batch operations sequence to describe the unit procedure, as well as particular processing costs, investment weights, required chemicals and resources. Hence, each equipment piece is associated to a unique and particular technology $\Lambda_{j} \subseteq \Lambda,\left|\Lambda_{j}\right|=1, \forall j \in U$. For instance, Fig. 7 shows a SEN with two technological alternatives $A$ and $B$ associated to equipment units $U_{1, A}$ and $U_{1, B}$ respectively. In order to consider several technologies in the same process stage, at least one equipment piece should be defined for each technological alternative, in order that their DAE systems can be differentiated according to Eq. 1. The technology $\lambda \in \Lambda_{j}$ associated to equipment unit $j \in U$ is represented by Boolean $V_{\lambda}^{j}$, which indicates whether such specification is selected $\left(V_{\lambda}^{j}=\right.$ true $)$ or not $\left(V_{\lambda}^{j}=\right.$ false $)$ and is related to the equipment Boolean $Y_{j}$ as follows:

$$
Y_{j} \Leftrightarrow V_{\lambda}^{j}, \quad \forall \lambda \in \Lambda_{j}, j \in U
$$

[Figure 7 about here.]

Selection of chemicals. The selection of chemicals like reactants, solvents, or catalysts $c \in C_{j}^{s}$ that are involved in the unit procedure of unit $j \in U$ is a synthesis decision represented by Boolean $S_{c}^{j}$, which indicates whether a chemical compound is selected $\left(S_{c}^{j}=\right.$ true $)$ or not $\left(S_{c}^{j}=\right.$ false). The SEN of Fig. 7 also illustrates an example with two potential reactants, solvents, or catalysts $c_{1}^{s}$ and $c_{2}^{s}$ in unit $U_{2}$. In contrast to the case of technological specification $\lambda \in \Lambda_{j}$, the use of alternative chemicals $c \in C_{j}^{s}$ does not affect the complete set of balance equations in the procedure model of unit $j \in U$ (Eq. 1), but only the set of parameters $p_{j}$ in such unit. Therefore, the process parameters $p_{j, c}$ associated to each potential alternative

$c \in C_{j}^{s}$ are included in the optimization problem as a function of the chemical selection Boolean $S_{c}^{j}$ as follows:

$$
\underset{c \in C_{j}^{s}}{\vee}\left[\begin{array}{c}
S_{c}^{j} \\
p_{j}=p_{j, c}
\end{array}\right], \forall j \in U
$$

\subsection{Synchronization}

The time axis for the models of batch units $j \in U$ that are selected (Eqs. 1 and 2 with $Y_{j}$ being true) should be moved along the time axis of the entire process at Level 0 according to their processing order, as illustrated in Fig. 4. This means that each batch unit model should be synchronized with the models of other plant elements, by relating the set of stages $K_{j}$ at Level 1 to specific stages from the set $L$ at Level 0 . With the purpose of leading the synchronization, task-unit assignment Booleans $W_{j, q}$ are defined for indicating the order $q \in Q$ of unit $j \in U$ with respect to other batch units. Once a unit is selected, this should be assigned one and only one processing order:

$$
Y_{j} \Leftrightarrow \underset{q \in Q}{\underline{\vee}} W_{j, q}, \quad \forall j \in U,
$$

and its operation should start in stage $l_{j, q}^{0} \in L_{j}^{0}$ associated to the $q$-th position with $W_{j, q}$ being true. This way, the relation between stage $k \in K_{j}$ at Level 1 and its corresponding stage $l \in L$ at Level 0 can be expressed as a function of the processing order $q \in Q$ of unit $j \in U$ through the function $l=k+l_{j, q}^{0}-1 \in L$. 
On this basis, the following variables are related across batch unit models at Level 1 and the general flow sheet model at Level 0:

- The starting time $t^{j, s}$ of unit $j \in U$ (Level 1) is calculated from the general starting time $t^{s}$ and duration $t_{l}$ of stages that precede $l_{j, q}^{0}$ (Level 0$)$ by:

$$
\underset{q \in Q}{\vee}\left[\begin{array}{c}
W_{j, q} \\
t^{j, s}=t^{s}+\sum_{l=1}^{l_{j, q}^{0}-1} t_{l}
\end{array}\right], \forall j \in U ;
$$

- The durations $t_{k}^{j}$ of stages $k \in K_{j}$ of unit $j \in U$ (Level 1 ) should be equal to the duration $t_{l}$ of corresponding stages $l \in L$ (Level 0$)$ by:

$$
\underset{q \in Q}{\vee}\left[t_{l}=t_{l-l_{j, q}^{0}+1}^{W_{j, q}}, \forall l \in\left\{l_{j, q}^{0}, \ldots,\left|K_{j}\right|+l_{j, q}^{0}-1\right\}\right], \forall j \in U ;
$$

- The input and output variables of unit $j \in U$ at Level $1\left(F_{m, k}^{j}(t)\right.$ and $x_{c, m, k}^{j}(t), \forall c \in C, \forall m \in$ $\left.M_{j}^{\text {in }} \cup M_{j}^{\text {out }}\right)$ should be equal to their analogous variables at Level $0\left(F_{n, l}(t)\right.$ and $x_{c, n, l}(t), \forall c \in C, \forall n \in$ $\left.N_{j}^{\text {in }} \cup N_{j}^{\text {out }}\right)$. These last ones should moreover be fixed to zero in the remaining non-synchronized stages and in the case that unit $j \in U$ is not selected. The resulting equation for every pair of equivalent flows at Levels 0 and $1(n, m) \in\left(N_{j}^{\text {in }}, M_{j}^{\text {in }}\right) \cup\left(N_{j}^{\text {out }}, M_{j}^{\text {out }}\right)$ reads as:

$$
\underset{q \in Q}{\vee}\left[\begin{array}{c}
W_{j, q} \\
F_{n, l}(t)=F_{m, l-l_{j, q}^{0}+1}^{j}(t), x_{c, n, l}(t)=x_{c, m, l-l_{j, q}^{0}+1}^{j}(t), \\
t \in[0,1], \forall l \in\left\{l_{j, q}^{0}, \ldots,\left|K_{j}\right|+l_{j, q}^{0}-1\right\}, \\
F_{n, l}(t)=0, x_{c, n, l}(t)=0, \\
t \in[0,1], \forall l \in L \backslash\left\{l_{j, q}^{0}, \ldots,\left|K_{j}\right|+l_{j, q}^{0}-1\right\}
\end{array}\right] \vee\left[\begin{array}{c}
\neg Y_{j} \\
F_{n, l}(t)=0, \\
x_{c, n, l}(t)=0, \\
t \in[0,1], \\
\forall l \in L
\end{array}\right], \forall j \in U .
$$

\subsection{Process stages}

Process stage selection. Logical variable $Z_{i}$ represents the selection of process stage $i \in P S$, which controls whether the input flow $n \in N_{i}^{i n}$ is processed or whether it is bypassed through pipeline $b \in N_{i}^{b}$ to the following task. This is represented in the problem superstructure through a splitter, like $S p_{3}$ in the example of Fig. 7. Since both alternatives are exclusive, the process stage selection reads as:

$$
\left[\begin{array}{c}
Z_{i} \\
F_{b, l}(t)=0
\end{array}\right] \underline{\vee}\left[\begin{array}{c}
\neg Z_{i} \\
F_{b, l}(t)=F_{n, l}(t)
\end{array}\right], t \in[0,1], \forall l \in L, \forall n \in N_{i}^{i n}, \forall b \in N_{i}^{b}, \forall i \in P S .
$$

Operating mode. Out of the set of allowed operating modes or configurations $\psi \in \Psi_{i}$ in a process stage $i \in P S$, which are associated to Boolean variables $X_{\psi}^{i}$, only one can be selected. Thus, the following proposition is defined:

$$
Z_{i} \Leftrightarrow \underset{\psi \in \Psi_{i}}{\underline{\vee}} X_{\psi}^{i}
$$

The principal purpose of configuration Booleans is to govern the selection of equipment items $j \in U$ represented by variable $Y_{j}$ and their processing order $q \in Q$ represented by variable $W_{j, q}$, by means of logical propositions that define each configuration. For example, operating modes $\Psi_{i}=\{\alpha, \beta, \pi, \sigma\}$ corresponding 
to single operation in $U_{1}$, single operation in $U_{2}$, parallel operation in $U_{1}$ and $U_{2}$, and series operation in $U_{1}$ followed by $U_{2}$ in the example of Fig. 1, read as:

$$
\begin{aligned}
& X_{\alpha}^{i} \Leftrightarrow W_{U_{1}, 1} \wedge \neg Y_{U_{2}}, \\
& X_{\beta}^{i} \Leftrightarrow W_{U_{2}, 1} \wedge \neg Y_{U_{1}}, \\
& X_{\pi}^{i} \Leftrightarrow W_{U_{1}, 1} \wedge W_{U_{2}, 1}, \\
& X_{\sigma}^{i} \Leftrightarrow W_{U_{1}, 1} \wedge W_{U_{2}, 2} .
\end{aligned}
$$

Additionally, configuration Booleans $X_{\psi}^{i}$ enforce a specific flow distribution for each operating mode, what is accomplished by determining the set of pipelines $n \in N_{i, \psi}^{0}$ whose flow rate is restricted to zero in each case:

$$
\underset{\psi \in \Psi_{i}}{\underline{\vee}}\left[\begin{array}{c}
X_{\psi}^{i} \\
F_{n, l}(t)=0, \quad t \in[0,1], \forall n \in N_{i, \psi}^{0}, \forall l \in L
\end{array}\right], \quad \forall i \in P S .
$$

\subsection{Plant elements at Level 0}

Active stages. The bypass strategy previously applied to batch units $j \in U$ (see Fig. 6) is also used at Level 0 to define the set of extra stages $L \backslash L^{a}$, out of the total number of stages $L^{\max }$, that should be dismissed in each structural alternative. This is because the sequence of batch operations, and therefore the set of active stages $L^{a}$ required to define the whole process, depends on the selected tasks, namely variables $Z_{i}$, and their configuration, namely variables $X_{\psi}^{i}$. Then, when a semi-continuous plant item $j \in J \backslash U$ is used, the set of stages $l \in L \backslash L^{a}$ that are not required at Level 0 are overridden according to Fig. 8, by forcing stage durations $t_{l}$ to zero and removing dynamic equations and constraints of Level 0 elements, as detailed next.

[Figure 8 about here.]

Models of plant elements with semi-continuous procedures. Time relations at Level 0 have the form:

$$
\begin{gathered}
T^{f}=\sum_{l \in L} t_{l}, \\
t^{\text {end }}=t^{s}+T^{f},
\end{gathered}
$$

where $T^{f}$ is the total time which starts at initial time $t^{s}$ of the process recipe, ends at final time $t^{\text {end }}$, and is divided in $L^{\max }$ intervals with duration $t_{l}$. The stage duration is zero in extra stages $l \in L \backslash L^{a}$, following

the bypass strategy represented in Fig. 8, whereas it is confined between lower $t^{\mathrm{L}}$ and upper $t^{\mathrm{U}}$ bounds in active ones $l \in L^{a}$ :

$$
\begin{aligned}
& t^{\mathrm{L}} \leq t_{l} \leq t^{\mathrm{U}}, \forall l \in\left\{1, \ldots,\left|L^{a}\right|\right\}, \\
& \text { bypass stages: } t_{l}=0, \quad \forall l \in\left\{\left|L^{a}\right|+1, \ldots, L^{\max }\right\} .
\end{aligned}
$$

Moreover, flow sheet balances are constructed at this level as $|L|$-stage models that relate the flow rate $F_{n, l}(t)$ and composition $x_{c, n, l}(t)$ of flows $n \in N$ and components $c \in C$ in connecting nodes, in every stage $l \in L$. Mixers $j \in M x$ have several input flows $n^{i n} \in N_{j}^{i n},\left|N_{j}^{i n}\right|>1$, and a single output flow 
$n^{\text {out }} \in N_{j}^{\text {out }},\left|N_{j}^{\text {out }}\right|=1$, and are described by:

$$
\begin{gathered}
\sum_{n^{i n} \in N_{j}^{i n}} F_{n^{i n}, l}(t)=F_{n^{\text {out }}, l}(t), t \in[0,1], \forall n^{\text {out }} \in N_{j}^{\text {out }}, \forall l \in L, \forall j \in M x, \\
\sum_{n^{i n} \in N_{j}^{\text {in }}} F_{n^{i n}, l}(t) x_{c, n^{\text {in }, l}}(t) \\
=F_{n^{\text {out }}, l}(t) x_{c, n^{\text {out }}, l}(t), t \in[0,1], \forall c \in C, \forall n^{\text {out }} \in N_{j}^{\text {out }}, \forall l \in L, \forall j \in M x,
\end{gathered}
$$

whereas splitters $j \in S p$ have a single input flow $n^{i n} \in N_{j}^{i n},\left|N_{j}^{i n}\right|=1$, and several output ones $n^{\text {out }} \in$ $N_{j}^{\text {out }},\left|N_{j}^{\text {out }}\right|>1$, and are described by:

$$
\begin{gathered}
F_{n^{\text {in }}, l}(t)=\sum_{n^{\text {out }} \in N_{j}^{\text {out }}} F_{n^{\text {out }}, l}(t), t \in[0,1], \forall n^{\text {in }} \in N_{j}^{\text {in }}, \forall l \in L, \forall j \in S p, \\
x_{c, n^{\text {in }}, l}(t)=x_{c, n^{\text {out }}, l}(t), t \in[0,1], \forall c \in C, \forall n^{\text {in }} \in N_{j}^{\text {in }}, \forall n^{\text {out }} \in N_{j}^{\text {out }}, \forall l \in L, \forall j \in S p .
\end{gathered}
$$

It is worth noting that it is not necessary to remove these equations in bypass stages, since they are cancelled by forcing flow rates to zero in previous Eqs. 9, 19 and 21.

Finally, storage units $j \in T$ are also defined at Level 0 by using $|L|$-stage models where all the equations are repeated in each active stage. Therefore, these models can be defined by the general functions $f_{j, l}^{d}, l_{j}^{d}$, $g_{j, l}^{d}, g_{j, l}^{d, e}, h_{j}^{d}$, and $m_{j, l}^{d}$ of Eq. 1 for storage tanks $j \in T$ and mathematical stages $l \in L^{a}$. Since these units operate continuously, $f_{j, l}^{d}=f_{j, l+1}^{d}, g_{j, l}^{d}=g_{j, l+1}^{d}$, and $g_{j, l}^{d, e}=g_{j, l+1}^{d, e}, \forall l \in\left\{1, \ldots,\left|L^{a}\right|-1\right\}$. The bypass strategy is then applied in two situations: First, if storage unit $j \in T$ is not selected, its complete model is deactivated like in the case of batch units $j \in U$. Second, the bypass method is required to differentiate active $L^{a}$ and bypass $L \backslash L^{a}$ stages according to Fig. 8, provided that this unit is selected. Summarizing, the model of storage tanks $j \in T$ is defined by:

$$
\begin{aligned}
& {\left[\begin{array}{c}
Y_{j} \\
f_{j, l}^{d}\left(\dot{z}_{j, l}(t), z_{j, l}(t), y_{j, l}(t), u_{j, l}^{d y n}(t), u^{\text {stat }}, u^{\text {int }}, p_{j}\right), t \in[0,1], \forall l \in\left\{1, \ldots,\left|L^{a}\right|\right\}, \\
l_{j}^{d}\left(\dot{z}_{j, 1}(0), z_{j, 1}(0)\right), \\
g_{j, l}^{d}\left(z_{j, l}(t), y_{j, l}(t), u_{j, l}^{d y n}(t), u^{s t a t}, u^{i n t}, p_{j}\right) \leq 0, \quad t \in[0,1], \forall l \in\left\{1, \ldots,\left|L^{a}\right|\right\}, \\
g_{j, l}^{d, e}\left(z_{j, l}(1), y_{j, l}(1), u_{j, l}^{d y n}(1), u^{\text {stat }}, u^{i n t}, p_{j}\right) \leq 0, \quad \forall l \in\left\{1, \ldots,\left|L^{a}\right|\right\}, \\
z_{j, l+1}(0)-m_{j, l}^{d}\left(z_{j, l}(1)\right)=0, \quad \forall l \in\left\{1, \ldots,\left|L^{a}\right|-1\right\}, \\
\gamma_{j}=h_{j}^{d}\left(z_{j,\left|L^{a}\right|}(1), y_{j,\left|L^{a}\right|}(1), u_{j,\left|L^{a}\right|}^{\text {dyn }}(1), u^{\text {stat }}, u^{\text {int }}, p_{j}\right), \\
\text { bypass stages: } B_{j}^{d}\left(\dot{z}_{j, l}(t), z_{j, l}(t), y_{j, l}(t), u_{j, l}^{d y n}(t), u^{\text {stat }}, u^{\text {int }}, \gamma_{j}, p_{j}\right)=0, \\
t \in[0,1], \forall l \in\left\{\left|L^{a}\right|+1, \ldots, L^{\text {max }}\right\}
\end{array}\right] \underline{v}} \\
& {\left[\begin{array}{c}
\neg Y_{j} \\
B_{j}^{d}\left(\dot{z}_{j, l}(t), z_{j, l}(t), y_{j, l}(t), u_{j, l}^{d y n}(t), u^{\text {stat }}, u^{i n t}, \gamma_{j}, p_{j}\right)=0, t \in[0,1], \forall l \in L
\end{array}\right], \forall j \in T,}
\end{aligned}
$$

where $z_{j, l}^{d}(t), z_{j, l}(t)$, and $u_{j, l}^{d y n}(t)$ are the time dependent differential, algebraic, and control variables in mathematical stage $l \in L, u^{\text {stat }}$ are time-invariant control variables, namely stage durations $t_{l}, u^{\text {int }}$ are integer decisions such as the storage tank size, $p_{j}$ are process parameters, and $\gamma_{j}$ are time-invariant variables which may contribute to the evaluation of the objective function or KPIs in unit $j \in T$. Finally, the maximum volume restriction should be also formulated, reading as:

$$
v_{l}^{j}(t) \leq \operatorname{Size}^{j}, \quad t \in[0,1], \forall l \in L, \forall j \in T,
$$


where $v_{l}^{j}(t)$ is the inventory volume of unit $j \in T$ in stage $l \in L$, which can not surpass the capacity Size $e^{j}$ of the tank. The decision on the selection of intermediate tanks is crucial for allowing the recirculation of intermediate flows - e.g. buffer tank $T_{11}^{r}$ in the example of Fig. 7 - as well as for defining more than one cycle time or batch size for different parts of the process.

Recirculation of intermediate material. The recirculation of an intermediate material to be used for processing a following batch is associated to flow $n \in N^{r}$. In particular, this decision is controlled by Boolean $R_{n}$, which determines the value of the recycle flow rate to be either between lower $F_{n}^{\mathcal{L}}$ and upper $F_{n}^{\mathcal{U}}$ bounds or cancelled:

$$
\left[\begin{array}{c}
R_{n} \\
F_{n}^{\mathcal{L}} \leq F_{n, l}(t) \leq F_{n}^{\mathcal{U}}, t \in[0,1], \forall l \in L
\end{array}\right] \underline{\vee}\left[\begin{array}{c}
\neg R_{n} \\
F_{n, l}(t)=0, t \in[0,1], \forall l \in L
\end{array}\right], \forall n \in N^{r} .
$$

Additionally, flow recirculation requires the installation of buffer tanks $j \in T_{n}^{r}$ in the superstructure, in order to store the intermediate material that is later supplied in a subsequent batch. The logical proposition reads as:

$$
R_{n} \Leftrightarrow Y_{j}, \quad \forall j \in T_{n}^{r}, \forall n \in N^{r} .
$$

In order to define mathematically the sequence of storage and use of intermediate material, it is necessary to set initial conditions $z_{j, l}^{d}(t=0)$ in the first stage $l=1$ of unit $T_{n}^{r}$ to be equal to the final conditions $z_{j, l}^{d}(t=1)$ in the last stage $l=L^{\max }$, what reads as:

$$
\left[z_{j, 1}^{d}(0)=z_{j, \mid L^{\max \mid}}^{R_{n}}(1), \forall j \in T_{n}^{r}\right], \forall n \in N^{r}
$$

\subsection{Batching}

The batching problem consists in the division of the total product demand into a number of batches with a specific production size. Typical approaches to solve scheduling problems first address the batching activity, and following solve the allocation, timing, and task sequencing sub-problems. The simultaneous solution of batch process development here tackled incorporates batching decisions into the integrated optimization problem by means of the following equation:

$$
N B_{p} \text { Batch }_{p}+\text { Shortfall }_{p} \geq \text { Demand }_{p}, \quad \forall p \in P,
$$

where $\operatorname{Demand}_{p}$ is the total demand of product $p \in P, N B_{p}$ represents the number of batches, Batch stands for the batch production size, and $S_{\text {hortfall }}$ is the unaccomplished demand.

\subsection{Objective function}

The problem is solved to minimize an objective function $\Phi$ :

$$
\underset{\substack{u_{k}^{d y n}(t), u^{\text {stat }}, u^{\text {int }}, u^{B \text { ool }}}}{\operatorname{minimize}} \Phi\left(z_{k}(t), y_{k}(t), u_{k}^{\text {dyn }}(t), u^{\text {stat }}, u^{\text {int }}, u^{\text {Bool }}, \gamma_{j}, p\right),
$$

according to the control variables $u_{k}^{d y n}(t)=\left\{F_{m, k}^{j}(t), i n t_{k}^{j}(t)\right\}, u^{\text {stat }}=\left\{t_{l}\right\}, u^{\text {int }}=\left\{N B_{p}\right.$, Size $e^{j}$, and $u^{\text {Bool }}=\left\{Z_{i}, Y_{j}, X_{\psi}^{i}, W_{j, q}, V_{\lambda}^{j}, S_{c}^{j}, R_{n}\right\}$. 
Degrees of freedom in the optimization model. The DOF of the system are defined as the number of decision variables subtracting the number of equations and predefined values. This parameter determines the number of practical control variables. In the particular case of Booleans $u^{B o o l}=\left\{Z_{i}, Y_{j}, X_{\psi}^{i}, W_{j, q}, V_{\lambda}^{j}, S_{c}^{j}, R_{n}\right\}$, the number of degrees of freedom is limited by logical propositions (Eqs. 4, 6, 9, 11, 12-15, and 26). As for dynamic control profiles of input and output stages $F_{m, k}^{j}(t) \subseteq u_{k}^{d y n}(t)$, these are interrelated through global balances in mixers and splitters (Eqs. 19 and 21). In this case, the evaluation of DOF is especially relevant between consecutive units, where input and output flow rates and compositions have to be synchronized and, therefore, can not behave as decision variables simultaneously. In fact, when the system is over-specified, their simultaneous consideration as control variables deteriorates the performance of the solution search procedure. Specifically, it is the flow distribution at Level 0 who determines which flow rates $F_{m, k}^{j}(t)$ of batch units $j \in U$ have to be equivalent or can behave as free decisions. As a result, the degrees of freedom related to flow rates $D O F_{i, \psi}$ are evaluated for each configuration $\psi \in \Psi_{i}$ in process stage $i \in P S$, as they depend on the total number of flow rates at Level $0\left|N_{i}\right|$, equations in splitters and mixers $\left|S p_{i} \cup M x_{i}\right|$, and predefined flow rates, namely flow restrictions $\left|N_{i, \psi}^{0}\right|$ for each configuration $\psi \in \Psi_{i}$ and input flows $\left|N_{i}^{f}\right|$ defined in preceding tasks. Overall, $D O F_{i, \psi}$ is defined as follows:

$$
\begin{aligned}
D O F_{i, \psi} & =\text { No. variables }- \text { No. equations }- \text { No. fixed variables } \\
& =\left|N_{i}\right|-\left|S p_{i} \cup M x_{i}\right|-\left|N_{i, \psi}^{0}\right|-\left|N_{i}^{f}\right| .
\end{aligned}
$$

To adjust the control variables to the degrees of freedom for each structural alternative, it is established that outflows of batch units are always free-decision variables:

$$
F_{m \text { out }, k}^{j}(t) \in u_{k}^{d y n}(t), t \in[0,1], \forall m^{\text {out }} \in M_{j}^{\text {out }}, \forall k \in O_{j}, \forall j \in U_{i}, \forall i \in P S,
$$

but only the inflows of the subset of batch units $D_{i, \psi} \subseteq U_{i}$ can be control variables in configuration $\psi \in \Psi_{i}$ of process stage $i \in P S$, where $\left|D_{i, \psi}\right|=D O F_{i, \psi}-\left|U_{i}\right|$ and $\left|U_{i}\right|$ represents the DOF that are removed by output flows in Eq. 31. The resulting equation reads as:

$$
\underset{\psi \in \Psi_{i}}{\stackrel{\vee}{\vee}}\left[\begin{array}{c}
X_{\psi}^{i} \\
F_{m^{i n}, k}^{j}(t) \in u_{k}^{d y n}(t), t \in[0,1], \forall m^{i n} \in M_{j}^{i n}, \forall k \in I_{j}, \forall j \in D_{i, \psi}
\end{array}\right], \forall i \in P S .
$$

\section{MLDO solution}

Several deterministic approaches to solve MLDO problems are available in the literature and current state-of-the-art [1, 61]. Specifically, a classical solution strategy based on the reformulation of the mixedlogic model into a mixed-integer one is followed in this work. This way, it is possible to exploit the advantages of mixed-logic modeling - e.g. the incorporation of previous knowledge and rules to optimization-based approaches- and yet to use well established MIDO solution strategies. Among the different classical methods, the direct-simultaneous strategy $[62,63]$ is based on a full discretization of the dynamic model, thus approximating process and control variable profiles into a series of finite points along the time horizon to obtain a MINLP formulation. The reader interested in alternative stochastic alternative stochastic and hybrid solution strategies is addressed to [64]. 


\subsection{MLDO reformulation}

Classical methods are based on the relaxation of the original MLDO model into MIDO one. For this, the following transformations are required. First, Boolean variables $u^{\text {Bool }} \in\{$ true, false $\}$ are replaced by binaries $u^{b i n} \in\{0,1\}$. Next, disjunctive equations are transformed into mixed-integer ones through binary multiplication, which is a further alternative to big-M [55] and Convex-Hull Reformulation (CHR) [65]. The method is based on the decomposition of differential $\dot{z}_{k}(t)$ and $z_{k}(t)$, algebraic $y_{k}(t)$, and timeinvariant $\gamma$ variables into contributions $\dot{z}_{k, i}(t), z_{k, i}(t), y_{k, i}(t)$, and $\gamma_{i}$ associated to each disjunctive term $i \in I D$ of disjunctive equations. Then, each contribution is multiplied by its binary variable $u_{i}^{b i n}$. For instance, function $f_{k, i}^{d}$ :

$$
\underset{i \in I D}{\vee}\left[\begin{array}{c}
u_{i}^{\text {Bool }} \\
f_{k, i}^{d}\left(\dot{z}_{k}(t), z_{k}(t), y_{k}(t), u_{k}^{d y n}(t), u^{\text {stat }}, u^{i n t}, \gamma, p\right)=0 \\
t \in[0,1], \forall k \in K
\end{array}\right]
$$

is transformed into:

$$
\begin{gathered}
f_{k, i}^{d}\left(\dot{z}_{k, i}(t), z_{k, i}(t), y_{k, i}(t), u_{k}^{d y n}(t), u^{\text {stat }}, u^{i n t}, \gamma_{i}, p\right)=0, t \in[0,1], \forall k \in K, i \in I D, \\
\dot{z}_{k}(t)=\sum_{i \in I D} \dot{z}_{k, i}(t) u_{i}^{b i n}, \\
z_{k}(t)=\sum_{i \in I D} z_{k, i}(t) u_{i}^{b i n}, y_{k}(t)=\sum_{i \in I D} y_{k, i}(t) u_{i}^{b i n}, \forall k \in K, \\
\gamma_{i}=\sum_{i \in I D} \gamma_{i} u_{i}^{b i n}, \quad \sum_{i \in I D} u_{i}^{b i n}=1 .
\end{gathered}
$$

Finally, logical propositions are expressed as linear constraints. This can be done systematically by formulating the Conjunctive Normal Form (CNF) of the original logical equations to obtain an expression like $C_{1} \wedge C_{2} \wedge \ldots \wedge C_{N}$, where $C_{n}$ are the clauses that must be true in the problem, related by "and" operators $(\wedge)$. This procedure involves the application of a series of pure logical operations and the transformation of the resulting true-clauses into equality or inequality equations [66, 55].

\subsection{MIDO solution}

The solution of the resulting MIDO problem is addressed through the full discretization of the model using the orthogonal collocation method. This was originally introduced by Cuthrell and Biegler [67] to solve optimal control problems with discontinuous control profiles, and renders attractive stability, symmetry, and accuracy properties $[67, \S 3.1]$. The strategy consists of dividing the time axis into a number of intervals -finite elements $e^{-}$and specific time points -collocation points $m^{-}$. The last ones are located by computing the roots of an orthogonal polynomial, like the shifted Legendre polynomial of order $M$. Then, the state and control variable profiles are approximated using monomial basis representations, such as Lagrange polynomials. In particular, an $M^{t h}$ order is established in the approximation of differential variables $z_{k}(t)$ in order to guarantee their continuity across neighboring finite elements, whereas $(M-1)^{t h}$ is defined for algebraic $y_{k}(t)$ and control $u_{k}^{d y n}(t)$ variables.

The originated MINLP problem can be solved through a number of search algorithms available in literature [68], which are principally based on enumeration or decomposition strategies. Many of them have 
been implemented in commercial software and can be accessed through modeling interfaces like GAMS [69]. Particularly, DICOPT solver is used in this study, which is based on Outer Approximation (OA) [54]. Being a decomposition-based strategy, the OA algorithm guarantees only local optima due to the existence of nonconvex terms in the model, e.g. bilinear functions associated to mixers. Therefore, it is necessary to repeat the optimization procedure for several initial feasible solutions (IFSs), pursuing to identify and avoid local solutions. The IFSs are computed in a preliminary step by optimizing the problem with constant control profiles and fixed configurations chosen randomly.

\section{Numerical example 1: Denbigh reaction system}

The numerical example presented in this section addresses the introduction of a specialty chemical into an already existing plant through the optimization of the integrated batch process development problem. The optimal solution is compared to standard predefined recipes in order to quantify the improvement potential of the proposed approach. Specifically, the process consists of a competitive reaction mechanism, the Denbigh reaction system [70]:

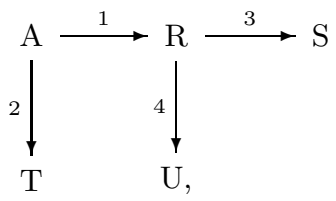

whose kinetic and thermodynamic data are available in Table S1 (see Supplementary Material). The SEN superstructure of the example corresponds to the reactor network of the Fig. 1, comprising two batch reactors $U_{1}$ and $U_{2}$ with a capacity of $1 \mathrm{~m}^{3}$. Since the investment in new equipment units and pipelines is initially not contemplated, the operating modes that can be selected are: operation in one single unit $U_{1}$ (configuration $\alpha$ ) or $U_{2}$ (configuration $\beta$ ), parallel in-phase operation in $U_{1}$ and $U_{2}$ (configuration $\pi$ ), or series operation in $U_{1}$ followed by $U_{2}$ (configuration $\sigma$ ). Batch unit procedures in the reactors are composed of load, hold, and unload operations and the control variables are the input and output flow rates and the reaction temperature.

\subsection{Optimization problem}

The production target is to fulfill a demand of $21 \mathrm{tn}$ of product $\mathrm{S}$ minimizing the raw material expenses within a time horizon of 144 hours. Additionally, a penalty is applied to the product shortfall. This way, the optimization problem is defined by:

$$
\underset{\substack{\text { minimize } \\ u_{k}^{\text {myn }}(t), u^{\text {stat }}, u^{\text {int }}, u^{\text {Bool }}}}{\operatorname{most}} \boldsymbol{A}+\text { Penalty },
$$


where $u_{k}^{d y n}(t)$ are input $F_{1, k}^{j}(t)$ and output $F_{2, k}^{j}(t)$ flow rates and reaction temperature $\theta_{k}^{j}(t)$ in stages $k \in\{1,2,3\}$ in $j \in\left\{U_{1}, U_{2}\right\}, u^{\text {stat }}$ is the duration $t_{l}$ of mathematical stages $l$, $u^{\text {int }}$ is the number of batches $N B_{\mathrm{S}}$, and $u^{B o o l}$ represent the Booleans for equipment units $Y_{j}$, task-unit assignments $W_{j, q}$, and configurations $X_{\psi}^{1}$. The first term of the objective function Cost $_{\mathrm{A}}$ stands for raw material expenses of the complete production campaign and is computed in base of a raw material price $\hat{p}_{\mathrm{A}}$ of $4.8 \mathrm{c} € / \mathrm{kg}$ (for further details, see Eqs. S15 and S20 in $\S$ S1 of the Supplementary Material), whereas the Penalty term represents the economic charge of unaccomplished demand, calculated considering twice the selling price $\hat{p}_{\mathrm{S}}$ of product $\mathrm{S}$, with a value of $43.1 \mathrm{c} € / \mathrm{kg}$ (see Eqs. S16 and S22). The complete MLDO model for solving this example is provided in the Supplementary Material, including the specifications of the already existing equipment items, which are incorporated into the optimization problem as operational constraints.

\subsection{Problem solution}

The MLDO problem is reformulated into a MINLP one using 32 finite elements and 3 collocation points in normalized Legendre roots, according to the direct-simultaneous strategy explained in $\S 5$. This is implemented in GAMS v.23.8.2 [69] and solved through the decomposition-based OA solver DICOPT [54], using CONOPT 3.15D and CPLEX 12.4 to handle the NLP and MILP sub-problems respectively. The optimization process uses four IFSs, which are first generated using constant profiles for the control variables $F_{1, k}^{j}(t), F_{2, k}^{j}(t)$, and $\theta_{k}^{j}(t)$ within each mathematical stage $k \in\{1,2,3\}$ and unit $j \in\left\{U_{1}, U_{2}\right\}$ and fixing the configuration Booleans $X_{\psi}^{1}=$ true for each of the four possible configurations $\psi \in\{\alpha, \beta, \pi, \sigma\}$. Next, the complete MINLP problem is solved with the four IFSs previously calculated and with no IFS. As seen in Table 1, which summarizes the features of the resulting MINLP models implemented in GAMS, the best solution is provided with IFS 4 .

[Table 1 about here.]

\subsection{Results and discussion}

The optimal solution of the integrated problem is compared with a fixed recipe that has a predefined production size of $300 \mathrm{~kg} / \mathrm{batch}$ of product $\mathrm{S}$ and requires 70 batches and a batch cycle time of $2.06 \mathrm{~h} / \mathrm{batch}$ to fulfill the demand of $21 \mathrm{tn}$ in its entirety within the time horizon of 144 hours. The operating mode is also fixed to configuration $\beta$, setting Boolean decisions $X_{\beta}^{1}, Y_{U_{2}}$, and $W_{U_{2}, 1}$ with a true value. Unit $U_{2}$ is defined to operate with maximum input and output flow rates $\left(7.7 \mathrm{~m}^{3} / \mathrm{h}\right)$ during load and unload operations and maximum reaction temperature $\left(110^{\circ} \mathrm{C}\right)$ to guarantee a feasible production. Besides, the optimization problem is solved for three cases with different DOF to track the effect of the structural and performance decisions:

- Dynamically optimal recipe: optimization of dynamic control profiles with fixed configuration $\beta$; 
- Structurally optimal recipe: optimization of structural decisions with constant profiles for control variables $F_{1,1}^{j}(t), F_{2,3}^{j}(t)$, and $\theta_{k}^{j}(t), k \in\{1,2,3\}, j \in\left\{U_{1}, U_{2}\right\}$;

- Optimal recipe i: simultaneous optimization of dynamic profiles and structural decisions according to the problem statement.

In all cases, the number of batches and the duration of batch operations are free decision variables.

Results obtained. The optimal operating mode for both optimizations that include structural decisions is series configuration $\sigma$, shown in Fig. 9a. This configuration activates and synchronizes the 3 -stage models associated to units $U_{1}$ and $U_{2}$, such that the unload operation of the first unit corresponds to the load of the second one. For the case of the optimal recipe i, the synchronization of batch operations is detailed in Fig. 9b, which illustrates the mathematical stages at Levels 1 and 0 for the production of one batch. As for the trajectories of dynamic control variables $F_{1,1}^{j}(t), F_{2,3}^{j}(t)$, and $\theta_{k}^{j}(t), k \in\{1,2,3\}, j \in\left\{U_{1}, U_{2}\right\}$, these are presented in Fig. 10(b1-c1,b2-c2), where it can be observed how all the control variable profiles range between their lower and upper bounds. The synchronization of transfer operations is illustrated through the correspondence between output flow rate $F_{3,2}^{U_{1}}$ from unit $U_{1}$ and input flow rate $F_{1,1}^{U_{2}}$ to unit $U_{2}$. Additionally, Fig. 10(b3-c3,b4-c4) shows the most relevant process variables, namely the reaction volume and molar compositions. All of them are compared to the trajectories of the fixed recipe in batch unit $U_{2}$ (Fig. 10(a1-a4)).

[Figure 9 about here.]

[Figure 10 about here.]

Improvement extent. The incentives to improve the process performance through the proposed modeling strategy are estimated by comparing the raw material savings obtained in each case with the fixed recipe. The results, summarized in Table 2 , show a decrease of the $12 \%$ in the consumption of A by optimizing the dynamic profiles with a predefined configuration $\beta$, whereas it is reduced as far as a $24 \%$ when qualitative decisions are considered as degrees of freedom with constant profiles. This improvement becomes a $25 \%$ when all decisions are optimized simultaneously in the optimal recipe i. The reduction of raw material expenses is related to the increase of the product selectivity, from 0.448 in the fixed recipe, to $0.507,0.588$, and 0.597 in the three optimal solutions (see Table 2). This is reflected in a value of the molar fraction of product $\mathrm{S}$ at the final time which is higher in optimal recipe (Fig. 10c4) than in the fixed recipe (Fig. 10a4). At the same time, the consumption of intermediate $R$ rises significantly in the optimal recipe, whereas the generation of byproducts like $\mathrm{T}$ is considerably lower.

[Table 2 about here.] 
Key decision variables. Essentially, the improvement of product selectivity is due to the optimization of the dynamic temperature profile and to the arrangement of the two reaction units in series (configuration $\sigma)$. On the one hand, the temperature profile in the optimal recipe starts at the lower bound $50^{\circ} \mathrm{C}$ in the unit procedure of $U_{1}$ (Fig. 10b1), in order to enhance the selectivity of $\mathrm{R}$ with respect to $\mathrm{T}$ by favoring reaction $1\left(E_{a, 1}=1000 \mathrm{kcal} / \mathrm{kmol}\right.$, see Table S1 in the Supplementary Material) with respect to reaction 2 $\left(E_{a, 2}=2580 \mathrm{kcal} / \mathrm{kmol}\right)$. The profile gradually increases to the upper bound of $80^{\circ} \mathrm{C}$ in $U_{1}$, thus favoring reaction $3\left(E_{a, 3}=1800 \mathrm{kcal} / \mathrm{kmol}\right)$ with respect to reaction $4\left(E_{a, 4}=1210 \mathrm{kcal} / \mathrm{kmol}\right)$ in order to improve the selectivity of the desired product $\mathrm{S}$ instead of $\mathrm{U}$. In the unit procedure of $U_{2}$, the temperature profile is risen even more (Fig. 10c1), reaching the upper bound of $110^{\circ} \mathrm{C}$ in this unit. On the other hand, production is restricted in all recipes by the maximum time horizon of $144 h$ (see Table 2) and by the available equipment capacities of $1 \mathrm{~m}^{3}$ in $U_{1}$ and $U_{2}$ (Fig. 10(a3-c3)). Therefore, series configuration $\sigma$ favors the process performance by means of enlarging the reaction volume.

Dynamic controls in transfer operations. In contrast, the use of dynamic control variables in transfer operations barely affects the solution. The feed-forward trajectories of flow rates in the optimal recipe are lead to their upper bound most of the time (Fig. 10(b2-c2)), obtaining profiles similar to the ones in the fixed recipe (Fig. 10a2). Thus, load and unload operations tend to be as brief as the pumping capacity permits. The dynamic feed-forward profile of reaction temperature is neither exploited in input and output stages and is kept constant (Fig. 10(b1-c1)). On the face of it, the optimization of a unique set-point in these stages would provide similar results.

Trade-offs among KPIs. Since heating costs are not included into the objective function, the interaction between selectivity of product $\mathrm{S}$ and processing expenses has not an effect in the optimal temperature profiles in this example. Regardless, processing costs associated to heat consumption also diminish (see Table 2) thanks to the reduction in energy consumption associated to side reactions 2 and 4 , which are endothermic. The rise of $\mathrm{S}$ selectivity also goes hand in hand to a reduction of reactant consumption and to a decrease in the total amount of reaction mixture required in the system. As a result, the heat required for reaching the reaction temperature is smaller and the optimal recipe benefits from a collateral reduction of heating costs of a 15\%. Besides, since new investments are not considered, null amortization values are computed in all recipes. Moreover, due to significant increase of occupation costs for using two reactors, the total profit is higher in the dynamically optimal recipe with fixed configuration $\beta$ than in the optimal recipe $\mathrm{i}$ with configuration $\sigma$, obtaining improvements of $11.9 \%$ and $6.5 \%$ respectively with regard to the profit of the fixed recipe. These results reflect the duplication of labor costs, such as unit cleaning and rental. 


\subsection{Adaptability potential}

In order to evaluate the potential of the proposed strategy to enhance plant flexibility and adaptability, the optimal recipe is solved for other decision criteria, namely the profit (case ii) and profitability (case iii) maximization, which additionally include product revenue $\left(\right.$ Revenue $\left._{\mathrm{S}}\right)$, processing cost $\left(\right.$ Cost $\left._{j, p}\right)$, occupational cost $\left(\operatorname{Cost}_{j, o}\right)$, and amortization cost $\left(\operatorname{Cost}_{j, a}\right)$ in unit $j$, and are defined through the minimization problems:

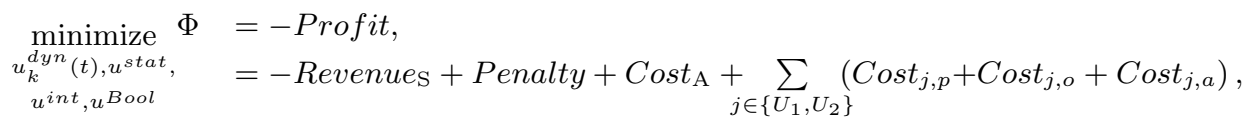

and

$$
\begin{aligned}
\underset{k}{u_{k} \text { minimize }(t), u^{\text {stat }},}, & =- \text { Profitability } \\
u^{\text {int }}, u_{\text {Bool }} & =-\frac{\text { Profit }}{T^{\text {total }}}
\end{aligned}
$$

considering the economic data detailed in Table S2 (see Supplementary Material). Moreover, the further process improvement that can be obtained with plant modifications is evaluated allowing the re-sizing of batch reactors for profit maximization (case iv). In this last case, the selection of equipment capacities larger than the original $1 \mathrm{~m}^{3}$ volume are treated as degrees of freedom, with their associated amortization.

The resulting KPIs for these cases are presented in Table 2. The most relevant variations between optimal recipes i-iv are the selected operating modes, batch processing times, number of batches, and temperature profiles, the last ones illustrated in Fig. 11. Besides, the optimal recipe with equipment capacity expansion involves the re-sizing of unit $U_{1}$ with a new volume of $3.5 \mathrm{~m}^{3}$. Overall, by including occupation costs into the objective function, series configuration $\sigma$ is replaced by parallel one $\pi$ because the simultaneous operation of $U_{1}$ and $U_{2}$ in configuration $\pi$ involves a that a lower number of batches is needed -e.g. 23 batches in case ii instead of 47 in case i. This way, the number of start-ups in each unit is reduced and the occupation costs, which have a term that depends on the number of start-ups, are reduced significantly in optimal recipes ii and iii (see Table 2 and Eqs. S2 and S18 in the Supplementary Material). As for the processing mode with capacity expansion of $U_{1}$, the optimal configuration is $\alpha$ with the single operation of unit $U_{1}$, preventing occupation costs in the smaller unit $U_{2}$.

[Figure 11 about here.]

Regarding the temperature profiles, the profit maximization leads to the maximum temperature of $80^{\circ} \mathrm{C}$ in unit $U_{1}$ and a compromise in $U_{2}$, operating at $94^{\circ} \mathrm{C}$ rather than at the upper bound of $110^{\circ} \mathrm{C}$. In contrast, profitability maximization also reaches the upper bound in unit $U_{2}$, since the processing time reduction is contemplated indirectly in the objective function in this case iii, hence the optimal solution takes into account the compromise between the economic gain and the reduction of the processing time. As a result, the processing time in optimal recipe iii achieves a lower value-almost three times less-compared to optimal recipe ii with profit maximization alone. Finally, the higher processing capacity in optimal recipe iv 
permits to operate at minimum temperature in recipes with much higher processing time and batch size (see Table 2).

Essentially, an improvement of the $23 \%$ in the total profit is recorded in optimal recipe ii compared to the use of the fixed recipe. This is related to a reduction of raw material, occupation, and processing costs, due to a higher S selectivity, lower energy consumption, and reduction in the number of unit start-ups. As for optimal recipe iii, it involves a rise of the $121 \%$ in the profitability objective with respect to the fixed recipe, even though this solution exhibits the highest total cost and the lowest selectivity of all the optimal solutions. Besides, the consideration of expanding the capacity of processing units in case iv hardly improves the total profit. The reason is that the original two-reactor plant was already equipped with the necessary capacity to fulfill the requested product demand, provided that these are properly arranged. However, the plant modification affects the cost distribution, being all of them reduced except for the amortization.

\section{Numerical example 2: Acrylic fiber production system}

This example addresses the development of an industrial-size batch copolymerization process to produce acrylic fiber with a specific composition and quality in a grassroots scenario. With this purpose, the presented modeling strategy is applied for constructing the integrated optimization model that simultaneously quantifies the interaction between process synthesis and plant allocation decisions like the selection of process stages, equipment technology, chemicals, and solvent recovery and reuse, among others.

The study of the integrated problem solution is motivated by the complex reaction mechanisms and physicochemical phenomena -for example, the auto-acceleration and the Trommsdorf effect- which determine the compromise between productivity and polymer quality. Then, the choice of processing conditions, such as reactor temperature and monomer feed rate trajectories, and processing times is crucial to determine the resulting polymer properties and production yield [71]. Additionally, there exist trade-offs between polymerization and downstream tasks, which also contribute to the achievements of overall economic and environmental production targets. Particularly, it has been reported the possibility to dismiss polymer-solvent separation stage when high conversions are achieved [72], the diverse environmental impact of organic and aqueous solvents depending on the molecular composition of the solvent [73], and the effect of cleaning technologies in polymerization processes [74].

The process for acrylic fiber production comprises a primary stage for bulk copolymer production and a secondary stage for transforming the copolymer into spun format, following the general process stages of the block diagram of Fig. 12 (see $\S \mathrm{S} 2$ of the Supplementary Material for further details). Besides, multiple operating modes can be considered for each particular task, determining the required processing units and their synchronization. Additionally, the following qualitative decisions can be made:

1. solution or suspension copolymerization in the reaction stage 1 , 
2. organic or aqueous solvent in solution copolymerization,

3. selection of separation stage 2 ,

4. recirculation of solvent recovered in separation stage 2 to be used in copolymerization stage 1 in a subsequent batch,

5. selection of washing and filtration stage 3 ,

6. recirculation of washing water in filtration stage 3 to copolymerization stage 1 ,

7. operating mode in process stage 7 : single unit $F_{71}$ or series $F_{71}$ followed by $F_{72}$,

8. operating mode in process stage 8: single unit $C_{81}$ or series $C_{81}$ followed by $C_{82}$,

9. recirculation of solvent recovered in separation stage 8 to copolymerization stage 1 or to repulping stage 4 .

The resulting SEN superstructure that represents all the potential process stages and structural alternatives for acrylic fiber production is presented in Fig. 13.

[Figure 12 about here.]

[Figure 13 about here.]

\subsection{Optimization problem}

The goal is to produce $5 \mathrm{tn}$ of acrylic fiber composed of $85 \%$ of acrylonitrile (AN) and $15 \%$ of vinyl acetate (VA) in bulk format minimizing the total operational and capital cost. Moreover, a maximum error of 0.025 in the composition should be fulfilled to guarantee polymer quality constraints. A single-product campaign is assumed to produce the total amount of final product in a time horizon of 144 hours. Particularly, the problem tackles the optimization of the batch production process and the batch plant according to the following objective function:

$$
\underset{\substack{u_{k}^{\text {dyn }}(t), u^{\text {stat }} \\ u^{\text {int }}, u^{\text {Bool }}}}{\operatorname{minimize}} \Phi=\text { Cost }_{\mathrm{M}_{1}}+\text { Cost }_{\mathrm{M}_{2}}+\text { Cost }_{\mathrm{I}}+\text { Cost }_{\mathrm{S}}+\text { Cost }_{a}+\text { Cost }_{p}+\text { Cost }_{\text {waste }},
$$

where $\mathrm{M}_{1}$ refers to $\mathrm{AN}$ monomer, $\mathrm{M}_{2}$ to VA monomer, I to initiator azobisisobutyronitrile (AIBN), and $\mathrm{S}$ to organic solvent dimethylformamide (DMF), aqueous solvent sodium thiocyanate (NaSCN(aq)), or suspension medium water. As for $u_{k}^{d y n}(t)$, it represents the profiles of input and output flow rates $\left(F_{i n 1, k}^{j}(t), F_{i n 2, k}^{j}(t)\right.$, $\left.F_{\text {out }, k}^{j}(t)\right)$ and cooling temperature $\left(\theta_{\text {cool, }, k}^{j}(t)\right)$ in the solution and suspension copolymerization reactors $j \in\left\{R_{11}, R_{12}\right\}$ which follow three operations $k \in\{1,2,3\}$ load, hold, and unload, as well as the heat $\left(Q_{\text {heat }, k}^{j}(t)\right)$ supplied in the evaporator $j \in\left\{E_{2}\right\}$ which follows three operations $k \in\{1,2,3\}$ load, distillate unload, and both fractions unload. $u^{\text {stat }}$ represents the composition of raw materials in the reactors' input stages $\left(c_{c, i n 1, k}^{j}, c \in\left\{\mathrm{M}_{1}, \mathrm{M}_{2}, \mathrm{I}\right\}, k \in\{1,2\}\right)$ and the duration of batch operations $\left(t_{l}, \forall l\right)$ and $u^{i n t}$ stands for the capacity $\left(S i z e^{j}\right)$ of processing units $j \in\left\{R_{11}, R_{12}, E_{2}\right\}$ and the number of batches $N B$. 
Regarding the logical decisions $u^{B o o l}$, these include the selection of separation stage $\left(Z_{i}, i \in\{2\}\right)$, the selection of solution or suspension technologies in copolymerization reactors $\left(V_{\lambda}^{j}, \lambda \in\{\right.$ solu, susp $\}, j \in$ $\left.\left\{R_{11}, R_{12}\right\}\right)$, the selection of organic or aqueous solvent in solution copolymerization reactor $\left(S_{c}^{j}, c \in\right.$ $\left.\{\mathrm{DMF}, \mathrm{NaSCN}(\mathrm{aq})\}, j \in\left\{R_{11}\right\}\right)$, and the selection of potential solvent recovery and reuse after the separation $\left(R_{2,1}\right)$. Such decisions are related to the selection of potential units $\left(Y_{j}\right)$, namely polymerization reactors $j \in\left\{R_{11}, R_{12}\right\}$, separation unit $j \in\left\{E_{2}\right\}$, and recirculation buffer tank $j \in\left\{T_{2}\right\}$. Finally, the multiple terms of the objective function $\Phi$ refer to: (i) the costs associated to raw material consumption, namely monomers $\mathrm{M}_{1}$ and $\mathrm{M}_{2}$, initiator $\mathrm{I}$, and solvent or suspension medium $\mathrm{S}\left(\right.$ Cost $_{\mathrm{M}_{1}}$, Cost $_{\mathrm{M}_{2}}$, Cost $_{\mathrm{I}}$, Cost $_{\mathrm{S}}$ ), (ii) equipment amortization $\left(\right.$ Cost $\left._{a}\right)$, (iii) total processing cost $\left(\right.$ Cost $\left._{p}\right)$, and (iv) costs associated to the waste disposal $\left(\right.$ Cost $\left._{\text {waste }}\right)$. The definition of these costs and the corresponding economic parameters can be found in $\S$ S2 of the Supplementary Material, (Eq. S38 and Table S11).

The MLDO model additionally includes disjunctive equations and logical propositions representing the abovementioned qualitative alternatives, as well as multistage models representing potential units. In particular, those process stages that have a with critical impact on the cost function are the copolymerization reaction - determining the selectivity and production yield- and the separation stage - governing the potential recovery of unreacted monomer, solvent, and suspension medium. Their process performance is represented by dynamic models with discrete events and is controlled by dynamic feed-forward control trajectories and batch operations duration. The model and process parameters are available in $\S$ S2 of the Supplementary Material.

\subsection{Problem solution}

The MLDO problem is solved through a direct-simultaneous strategy using 8 finite elements and 3 collocation points in normalized Legendre roots in the full discretization. Piece-wise constant functions are used to define the profiles of control variables. The resulting MINLP is solved with the OA solver DICOPT in GAMS optimization framework, using CONOPT and CPLEX solvers in the NLP and MILP sub-problems respectively. Additionally, given the low influence of dynamic profiles in transfer operations observed in example 1 , the dynamic models are approximated in load and unload operations, namely stages $k \in\{1,3\}$ in reactors $j \in\left\{R_{11}, R_{12}\right\}$ and evaporator $j \in\left\{E_{2}\right\}$.

Even though the resulting MINLP model is moderate in size, as it can be observed in Table 3, the presence of non-linear terms in a mixed-integer problem can lead to infeasible or sub-optimal solutions. To avoid this difficulty, the problem is solved in two steps. First, several subsystems are defined by fixing Boolean variables and reducing the set of discrete decisions to the number of batches and the equipment size. This way, the combinatorial part of the problem is limited. Next, the subsystem solutions serve as initial feasible points (IFSs) in the optimization of the integrated MINLP problem with all the degrees of freedom, in order to start the search procedure from different initial points. The subsystems are defined by 
fixing the selected technology and solvent as follows:

(1) solution polymerization and organic solvent, i.e. $V_{\text {solu }}^{R_{11}}, S_{\mathrm{DMF}}^{R_{11}}=$ true,

(2) solution polymerization and aqueous solvent, i.e. $V_{\text {solu }}^{R_{11}}, S_{\mathrm{NaSCN}(\mathrm{aq})}^{R_{11}}=$ true,

(3) suspension polymerization, i.e. $V_{\text {susp }}^{R_{12}}=$ true.

Each polymerization technology and solvent combination is evaluated without and with recirculation of the distillate fraction of the evaporator $E_{2}\left(R_{2,1}=\right.$ false in subsystems $1 \mathrm{a}, 2 \mathrm{a}$, $3 \mathrm{a}$, and $R_{2,1}=t r u e$ in $1 \mathrm{~b}$, $2 \mathrm{~b}, 3 \mathrm{~b})$. Additionally, the subsystem without separation stage is studied for solution polymerization with organic solvent $\left(Z_{2}=\right.$ false in subsystem $\left.3 \mathrm{c}\right)$. The remaining Booleans are computed through the logical propositions of the problem. Overall, seven sets of fixed logical variables are defined to generate IFSs. The associated MINLP model features are summarized in Table 3 for some them.

[Table 3 about here.]

\subsection{Results and discussion}

Trade-off among process stages. The optimal objective function of each subsystem is presented in the diagram of Fig. 14 in front of the optimal solution of the integrated MINLP problem for comparison. It is worth noting that the optimal solution among the three polymerization and solvent alternatives 1 , 2, or 3 in the reaction stage depends on decisions associated to other process stages, namely the recirculation selection. Suspension polymerization (subsystem 3a) is chosen when the recirculation of the distillate is not allowed, whereas solution polymerization with organic solvent (subsystem 1b) is the best alternative otherwise. At the same time, the selection of separation stage absolutely depends on decisions made in the reaction, like the polymerization temperature and monomer dosage, which determine the achieved monomer conversion, and the solvent selected. The only possibility for not requiring separation is solution polymerization with DMF and a minimum conversion (subsystem 1c). The proposed modeling approach integrates all these degrees of freedom and permits their simultaneous evaluation to obtain the optimal solution considering all the trade-offs among process stages.

[Figure 14 about here.]

Cost balance. The KPIs and cost contributions of the integrated and subsystems solutions are summarized in Table 4. Essentially, the heaviest cost weights are related to equipment amortization and raw material, being considerably higher than the expenses associated to cooling water in the reaction and heating in the separation. Although the combined costs of monomers $\mathrm{M}_{1}$ and $\mathrm{M}_{2}$ are the most notorious as it could be expected, the selection of organic and aqueous solvent in $R_{11}$ entails a large expenditure as well. Therefore, the possibility of recirculating solvent in subsystems $1 \mathrm{~b}$ and $2 \mathrm{~b}$ involve important raw material savings without detriment of the reaction effectiveness. However, despite raw material savings with recirculation, 
the optimal solution gives priority to the total cost minimization by means of eliminating the equipment investment associated to the evaporator $E_{2}$ and the recirculation storage $T_{2}$.

System adaptability. Anecdotally, comparing these results with preliminary calculations with fixed batch size, it is worth mentioning that the results here presented provide solutions with more similar values of the objective function for the different subsystems thanks to the consideration of the number of batches as a degree of freedom. However, this similarity does not represent homogeneity in the optimal solution of each subsystem, but adaptability of the model to provide favorable solutions by evaluating the decisions trade-offs. Actually, previous research on flexible plant design [24] has proved the enhancement of batch plant flexibility by combining process synthesis decisions, like dynamic control profiles, with plant allocation decisions, like batch sizing.

[Table 4 about here.]

System response. The optimal processing scheme corresponds to solution polymerization without separation, illustrated in Fig. 15, and is characterized by the control and processing variable profiles shown in Fig. 16. The optimal temperature trajectory in the polymerization reactor follows a monotonically increasing function, whereas the $\mathrm{M}_{1}$ dosage features a local maximum in the second half of the process that guarantees the desired polymer composition of $85 \%$ of $\mathrm{M}_{1}$. Thanks to the dosage profile and the large task duration (i.e. $18.4 h$ ) the process can be driven with a limited excess of monomers and fulfill the minimum monomer conversion of the $75 \%$ required in this process structure.

This solution is compared to the optimization of the same problem, halving the unitary amortization costs. In this case, the optimal solution is suspension polymerization with recirculation $\left(i . e\right.$. $V_{s u s p}^{R_{12}}=$ true, $R_{2,1}=$ true), since the raw material savings is prioritized in front of amortization cost reduction, which is smaller than in the original economic scenario. The control and processing variable profiles, illustrated in Fig. 17, are characterized by raw material and recirculation input flows in the first load operation in $R_{12}$ and a consequent reduction of the monomer dosage along the second stage. Additionally, there is a larger excess of monomer $\mathrm{M}_{1}$ for guaranteeing the desired composition, which is totally recovered in the distillate fraction in $E_{2}$. Even though the polymerization time is shorter in this case, with a cycle time of $9.1 h$, the solution still exhibits a polymerization reaction much longer than the separation. Therefore, the consideration of more polymerization reactors with a parallel out-of-phase configuration would be challenging to reduce the cycle time in case of desiring larger productions.

[Figure 15 about here.]

[Figure 16 about here.]

[Figure 17 about here.] 
To conclude, the major strength of the proposed methodology is the holistic evaluation of the decision criteria. In particular, the optimization model considers the trade-offs between the various process stages within the complete process, as well as the interactions between synthesis and allocation degrees of freedom. Additionally, it allows the analysis of processing alternatives according to multiple points of view, like processing, economic, or sustainable production policies. The primary copolymerization stage for acrylic fiber production has been successfully solved with regard to the isolated solution of particular structures. To fully exploit the methodology, it would be challenging to consider the simultaneous solution of the primary and secondary polymerization processes. Additionally, further degrees of freedom have been detected, which could be incorporated into the model, as it is the use of several reactors and their arrangement in parallel out-of-phase configuration to allow the reduction of the cycle time for pursuing greater demands in a limited time horizon.

\section{Conclusions and future directions}

This work introduces a modeling strategy to tackle batch process development and improvement in production scale, pursuing optimal solutions that enhance process efficiency and guarantee the best utilization of available equipment and new investments. With this purpose, multiple degrees of freedom associated to process synthesis and plant allocation sub-problems are solved simultaneously, taking into account the plant design and retrofit. The principal novelty of this strategy is the synchronization of unit procedures as a function of alternative processing schemes in an integrated model. From a modeling perspective, great progress has been made through the combination of hybrid discrete/continuous models, dynamic optimization, and mixed-logic modeling in a formulation that can be handled by current optimization tools. Overall, the resulting MLDO model handles the problem complexity by means of mixed-logic equations, while its reformulation into a MINLP problem enables its optimization through well-established search algorithms.

The significant improvement of plant adaptability gained through the presented approach also justifies and stimulates the research in this direction, especially having proved that the complex mathematical implications and the risk of obtaining mathematically intractable problems can be overcome with appropriated solution strategies like the use of initial feasible solutions and the careful definition of variable bounds. The promising results of the numerical example show improvements between the $23 \%$ and $121 \%$ in the objective function compared to the use of fixed recipes in all the optimization scenarios i-iv of the Denbigh example. In addition, the agile evaluation of diverse objective functions proves the potential of the proposed strategy to adapt master recipes according to in-time needs, as they could be changes in economic scenarios or decision criteria, and to analyze the system response in front of uncertain parameters.

This strategy also enables the quantification of interactions between synthesis and allocation sub-problems. This way, a greater influence on structural decisions with constant set-points optimization is identified in 
the Denbigh example in comparison to dynamic profiles optimization with fixed equipment configuration, rendering improvements over the fixed recipe of the $24 \%$ and the $12 \%$ respectively. In contrast, the consideration of dynamic flow rate profiles in material transfer operations has a small influence, since most of the transfer stages in the obtained optimal solutions are characterized by constant profiles along time in the numerical cases tested.

Finally, the trade-offs among decisions in neighboring process stages are studied in the acrylic fiber example, showing the influence of the distillate recirculation in separation stage over reaction stage decisions like the technological alternative and solvent selection. Likewise, reaction stage decisions like temperature and dosage profiles, raw monomer concentration, and processing times, which define the monomer conversion, determine the possibility of not selecting separation stage. The great advantage of the presented integrated strategy is its potential to assess all processing alternatives simultaneously and avoid enumeration methods that could miss optimal solutions.

Future work can be focused on the incorporation of additional degrees of freedom in the problem, like out-of-phase parallel operation and multi-product/multi-purpose production campaigns. The modular representation of unit procedures in the presented strategy should allow the direct extension of the formulation to achieve this purpose. Equally, the refinement of solution strategies will be relevant to face problems with a larger number of process stages and products and to meet global optimality. In this regard, the study of hybrid approaches that combine deterministic solvers with evolutionary algorithms is proposed as much as global deterministic solvers.

\section{Acknowledgments}

The authors would like to thank the financial support received from the Spanish Ministry of Economy and Competitiveness and the European Regional Development Fund (research project SIGERA DPI201237154-C02-01 and FPU Research Fellowship).

\section{Supplementary Material}

[Model of the Denbigh case study (§S1). Model of the acrylic fiber case study (§ S2)]. This material is available free of charge via the Internet at http://www.sciencedirect.com.

\section{Notation}

\section{General sets}

ID Set of disjunctive terms

\section{General variables}




$\begin{array}{ll}z_{k}(t) & \text { Differential process variables in mathematical stage } k \\ y_{k}(t) & \text { Algebraic process variables in mathematical stage } k \\ u_{k}^{d y n}(t) & \text { Dynamic control variables in mathematical stage } k \\ u^{\text {stat }} & \text { Time-invariant or static continuous control variables } \\ u^{\text {int }} & \text { Integer control variables } \\ u^{\text {Bool }} & \text { Logical or Booleans decisions } \\ u^{\text {bin }} & \text { Binary decisions } \\ \gamma & \text { Algebraic time-invariant variables } \\ p & \text { Process parameters }\end{array}$

\section{General functions}

A Matrix of the semi-explicit DAE system of differentiation index 1 at most

$f \quad$ DAE system

$g \quad$ Path constraints

$g^{e} \quad$ End-point constraints

$h \quad$ Algebraic equations evaluated at the final time

$l \quad$ Set of relations that define initial conditions

$m \quad$ Stage-to-stage continuity between consecutive mathematical stages

$f^{d} \quad$ DAE system in disjunctive equations

$g^{d} \quad$ Path constraints in disjunctive equations

$g^{d, e} \quad$ End-point constraints in disjunctive equations

$h^{d} \quad$ Algebraic equations evaluated at the final time in disjunctive equations

$l^{d} \quad$ Set of relations that define initial conditions in disjunctive equations

$m^{d} \quad$ Stage-to-stage continuity between consecutive mathematical stages in disjunctive equations

$B^{d} \quad$ Equations system to define bypass stages in disjunctive equations

$\Omega \quad$ Logical propositions

$\Phi \quad$ Objective function

\section{Problem sets}

$\Lambda \quad$ Set of technological specifications

$\Lambda_{j} \subseteq \Lambda \quad$ Technological specification of unit $j \in U,\left|\Lambda_{j}\right|=1, \forall j \in U$

$\Psi_{i} \quad$ Set of operating modes in task $i \in P S$

$C \quad$ Set of chemical compounds involved into the process

$C_{j}^{s} \subseteq C \quad$ Subset of potential reactants, solvents, or catalysts in unit $j \in U$

$D_{i, \psi} \subseteq U_{i}$ Subset of batch units $U_{i}$ in task $i \in P S$ whose input flow rate is a control variable in configuration $\psi \in \Psi_{i}$. It is defined such that $\left|D_{i, \psi}\right|=D O F_{i, \psi}-\left|U_{i}\right|$, where $\left|U_{i}\right|$ represents DOF removed by output flow rates

$I_{j} \subseteq K_{j} \quad$ Subset of input stages for unit $j \in U$ at Level 1 
$J \quad$ Set of all existing and potential equipment pieces, $J=U \cup T \cup S p \cup M x$

$J_{i} \subseteq J \quad$ Subset of equipment pieces within potential task $i \in P S$

$K_{j} \quad$ Set of stages for unit $j \in U$ at Level 1

$L \quad$ Set of potential stages at Level $0, L=\left\{1, \ldots, L^{\max }\right\}$

$L^{a} \subseteq L \quad$ Subset of active stages at Level 0

$L_{j}^{0} \subseteq L \quad$ Subset of stages at Level 0 where unit $j \in U$ can start its operation, $L_{j}^{0}=\left\{1,\left|K_{j^{\prime}}\right|-\left|O_{j^{\prime}}\right|+1 \mid j^{\prime} \neq j\right.$, $\left.j^{\prime} \in U\right\}$

$M_{j} \quad$ Set of pipelines for unit $j \in U$ at Level 1

$M_{j}^{i n} \subseteq M_{j}$ Subset of input pipelines to unit $j \in U$ at Level 1

$M_{j}^{\text {out }} \subseteq M_{j}$ Subset of output pipelines from unit $j \in U$ at Level 1

$M x \quad$ Set of existing and potential mixers

$N \quad$ Set of pipelines at Level 0

$N^{r} \subseteq N \quad$ Subset of pipelines at Level 0 for recirculation

$N_{i} \subseteq N \quad$ Subset of pipelines at Level 0 for task $i \in P S$

$N_{i}^{b} \subseteq N_{i} \quad$ Bypass pipeline for process stage $i \in P S,\left|N_{i}^{b}\right|=1$

$N_{i}^{f} \subseteq N \quad$ Subset of pipelines at Level 0 for task $i \in P S$ whose flow rate is fixed by the preceding task, $\left|N_{i}^{f}\right|=1$ except for process stages preceded by a buffer $j \in T$

$N_{i}^{i n} \subseteq N_{i}$ Subset of input pipelines to process stage $i \in P S$

$N_{i}^{\text {out }} \subseteq N_{i}$ Subset of output pipelines to process stage $i \in P S$

$N_{i, \psi}^{0} \subseteq N$ Subset of pipelines at Level 0 whose flow rate is restricted to zero in configuration $\psi \in \Psi_{i}$ of task $i \in P S$

$N_{j}^{i n} \subset N \quad$ Subset of input pipelines to unit $j \in J$ at Level 0

$N_{j}^{\text {out }} \subset N$ Subset of output pipelines from unit $j \in J$ at Level 0

$O_{j} \subseteq K_{j} \quad$ Subset of output stages for unit $j \in U$ at Level 1

$P \subset C \quad$ Subset of desired products

$P S \quad$ Set of potential process stages or tasks

$Q \quad$ Set of ordered positions that can be assumed by unit procedures of $j \in U$

$S p \quad$ Set of existing and potential splitters

$T \quad$ Set of existing and potential storage tanks

$T_{n}^{r} \subseteq T \quad$ Buffer tank for potential recirculation of flow $n \in N^{r},\left|T_{n}^{r}\right|=1, \forall n \in N^{r}$

$U \quad$ Set of existing and potential batch units

$U_{i} \subseteq U \quad$ Subset of existing and potential batch units in process stage $i \in P S$

\section{Problem parameters}

$D m_{p} \quad$ Demand of product $p \in P$

$D O F_{i, \psi} \quad$ Degrees of freedom with regard to the flow rates at Level 0 at process stage $i \in P S$, according to each configuration $\psi \in \Psi_{i}$ 
$l_{j, q}^{0} \quad$ Starting stage of unit $j \in U$ when the task-unit Boolean $W_{j, q}$ is $\operatorname{true}, l_{j, q}^{0}=q$-th element of the ascending sort of $L_{j}^{0}$ of unit $j \in U$

$L^{\max } \quad$ Maximum number of stages at Level 0

$p_{j, c} \quad$ Values for the set of process parameters $p_{j}$ in unit $j \in U$ when potential chemical alternative $c \in C_{j}^{s}$ is selected

Problem variables (* Control variables)

$v_{k}^{j}(t) \quad$ Material volume of unit $j \in U$ in stage $k \in K_{j}$ at Level 1 and of unit $j \in J \backslash U$ in stage $k \in L$ at Level 0

Batch $_{p} \quad$ Production size associated to each batch of product $p \in P$

$F_{m, k}^{j}(t)\left(^{*}\right)$ Flow rate for input or output pipeline $m \in M_{j}^{i n} \cup M_{j}^{\text {out }}$ in stage $k \in K_{j}$ of unit $j \in U$ at Level 1

$F_{n, l}(t) \quad$ Flow rate for every pipeline $n \in N$ in stage $l \in L$ at Level 0

$i n t_{k}^{j}(t)\left(^{*}\right)$ Internal control variable of unit $j \in U$ at Level 1 in stage $k \in K_{j}$ and of unit $j \in J \backslash U$ at Level 0 in stage $k \in L$

$N B_{p}(*) \quad$ Number of batches of product $p \in P$

$R_{n}\left(^{*}\right) \quad$ Recirculation Boolean for intermediate flow recycle $n \in N^{r}$

$S_{c}^{j}\left(^{*}\right) \quad$ Compound Boolean for reactant, solvent, or catalyst $c \in C_{j}^{s}$ in unit $j \in U$

Shortfall $p$ Unaccomplished demand of product $p \in P$

Size $\left.e^{*}\right) \quad$ Capacity of unit $j \in U \cup T$ with a discrete value

$T^{f} \quad$ Total time at Level 0

$t^{s} \quad$ Starting time at Level 0

$t^{\text {end }} \quad$ Final time at Level 0

$t^{j, e n d} \quad$ Final time of unit $j \in U$ at Level 1

$T^{j, f} \quad$ Total time of unit $j \in U$ model at Level 1

$t^{j, s} \quad$ Starting time of unit $j \in U$ at Level 1

$t_{k}^{j} \quad$ Duration of stage $k \in K_{j}$ of unit $j \in U$ at Level 1

$t_{l}(*) \quad$ Duration of stage $l \in L$ at Level 0

$V_{\lambda}^{j}(*) \quad$ Technology Boolean for specification $\lambda \in \Lambda_{j}$ in processing unit $j \in U$

$W_{j, q}\left(^{*}\right) \quad$ Task-unit assignment Boolean for processing order $q \in Q$ in unit $j \in U$

$X_{\psi}^{i}\left({ }^{*}\right) \quad$ Configuration Boolean for alternative $\psi \in \Psi_{i}$ in process stage $i \in P S$

$x_{c, m, k}^{j}(t) \quad$ Flow composition of compound $c \in C$ for input or output pipeline $m \in M_{j}^{\text {in }} \cup M_{j}^{\text {out }}$ in stage $k \in K_{j}$ of unit $j \in U$ at Level 1

$x_{c, n, l}(t) \quad$ Flow composition of compound $c \in C$ for every pipeline $n \in N$ and stage $l \in L$ at Level 0

$Y_{j}\left(^{*} \quad\right.$ Equipment Boolean for processing or storage unit $j \in U \cup T$

$Z_{i}\left(^{*}\right) \quad$ Process stage Boolean for task $i \in P S$ 


\section{References}

[1] J. Oldenburg, W. Marquardt, Disjunctive modeling for optimal control of hybrid systems, Computers \& Chemical Engineering 32 (10) (2008) 2346 - 2364, ISSN 0098-1354.

[2] D. W. T. Rippin, Simulation of single- and multiproduct batch chemical plants for optimal design and operation, Computers \& Chemical Engineering 7 (3) (1983) 137-156.

[3] G. Stephanopoulos, G. V. Reklaitis, Process systems engineering: From Solvay to modern bio- and nanotechnology: A history of development, successes and prospects for the future, Chemical Engineering Science 66 (19) (2011) 4272-4306.

[4] A. A. Linninger, E. Stephanopoulos, S. A. Ali, C. Han, G. Stephanopoulos, Generation and assessment of batch processes with ecological considerations, Computers \& Chemical Engineering 19, Supplement 1 (S1) (1995) 7-13.

[5] B. S. Ahmad, Synthesis of Batch Processes with Integrated Solvent Recovery, Ph.D. thesis, Massachusetts Institute of Technology, 1997.

[6] P. I. Barton, B. S. Ahmad, W. Cheong, J. Tolsma, Synthesis of batch processes with integrated solvent recovery, in: S. K. Sikdar, U. Diwekar (Eds.), Tools and Methods for Pollution Prevention, vol. 62, Kluwer Academic Publishers, Dordrecht, 205-231, 1999.

[7] S. A. Ali, Synthesis of batch processing schemes for the production of pharmaceuticals and specialty chemicals, Ph.D. thesis, Massachusetts Institute of Technology, 1999.

[8] M. Sharif, N. J. Samsatli, N. Shah, Abstract design in the development of pharmaceutical processes, in: S. Pierucci (Ed.), European Symposium on Computer Aided Process Engineering-10, vol. 8 of Computer Aided Chemical Engineering, Elsevier, 685-690, 2000.

[9] I. Papaeconomou, Integration of Synthesis and Operational Design of Batch Processes, Ph.D. thesis, Department of Chemical Engineering. Technical University of Denmark, 2005.

[10] O. A. Iribarren, Batch Chemical Processes Design, Ph.D. thesis, University of Massachusetts, 1985.

[11] O. Iribarren, M. Malone, H. Salomone, A Heuristic Approach for the Design of Hybrid Batch-Continuous Processes, Chemical Engineering Research \& Design 72(A3) (1994) 295-306.

[12] L. Cavin, U. Fischer, F. Glover, K. Hungerbühler, Multi-objective process design in multi-purpose batch plants using a Tabu Search optimization algorithm, Computers \& Chemical Engineering 28 (4) (2004) 459-478.

[13] A. Mosat, L. Cavin, U. Fischer, K. Hungerbühler, Multiobjective optimization of multipurpose batch plants using superequipment class concept, Computers \& Chemical Engineering 32 (3) (2008) 512-529.

[14] B. Srinivasan, S. Palanki, D. Bonvin, Dynamic optimization of batch processes - I. Characterization of the nominal solution, Computers \& Chemical Engineering 27 (1) (2003) 1-26.

[15] T. Binder, L. Blank, H. G. Bock, R. Bulirsch, W. Dahmen, M. Diehl, T. Kronseder, W. Marquardt, J. P. Schlöder, O. von Stryk, Introduction to model based optimization of chemical processes on moving horizons, Springer-Verlag, Berlin, 295339, 2001.

[16] V. S. Vassiliadis, R. W. H. Sargent, C. C. Pantelides, Solution of a Class of Multistage Dynamic Optimization Problems. 1. Problems without Path Constraints, Industrial \& Engineering Chemistry Research 33 (9) (1994) 2111-2122.

[17] J. Oldenburg, W. Marquardt, D. Heinz, D. B. Leineweber, Mixed-logic dynamic optimization applied to batch distillation process design, AIChE Journal 49 (11) (2003) 2900-2917.

[18] S. Jain, J.-K. Kim, R. Smith, Process Synthesis of Batch Distillation Systems, Industrial \& Engineering Chemistry Research 52 (24) (2013) 8272-8288.

[19] G. Reklaitis, Progress and issues in computer-aided batch process design, in: 3rd International Conference on Foundations of Computer-Aided Process Design, Snowmass, Colorado, July 09-14, 241-275, 1989.

[20] A. P. Barbosa-Póvoa, A critical review on the design and retrofit of batch plants, Computers \& Chemical Engineering 31 (7) (2007) 833-855. 
[21] J. D. Robinson, Y. R. Loonkar, Minimizing capital investment for multi-product batch-plants, Process Tehnology 17 (11) (1972) 861.

[22] R. Gani, I. Papaeconomou, Conceptual Design and Synthesis of Batch Processes, in: Chemical Industries, CRC Press, 43-82, 2005.

[23] P. I. Barton, R. J. Allgor, W. F. Feehery, S. Galan, Dynamic optimization in a discontinuous world, Industrial \& Engineering Chemistry Research 37 (3) (1998) 966-981.

[24] M. Moreno-Benito, A. Espuña, L. Puigjaner, Flexible Batch Process and Plant Design Using Mixed-Logic Dynamic Optimization: Single-Product Plants, Industrial \& Engineering Chemistry Research 0 (0) (2014) -.

[25] I. Grossmann, A. Westerberg, L. Biegler, Retrofit design of processes, in: G. Reklaitis, H. Spriggs (Eds.), Foundations of Computer Aided Process Operations. Proceedings of the First International Conference, ISBN 0444 98925 0, 403-42, 1987.

[26] M. S. Charalambides, N. Shah, C. C. Pantelides, Synthesis of batch reaction/distillation processes using detailed dynamic models, Computers \& Chemical Engineering 19 (S1) (1995) 167 - 174.

[27] M. Sharif, N. Shah, C. C. Pantelides, Design of integrated batch processes with discrete and continuous equipment sizes, Computers \& Chemical Engineering 23 (Supp.) (1999) S117-S120.

[28] R. Allgor, L. Evans, P. Barton, Screening models for batch process development Part I. Design targets for reaction/distillation networks, Chemical Engineering Science 54 (19) (1999) 4145-4164.

[29] O. A. Iribarren, J. M. Montagna, A. R. Vecchietti, B. Andrews, J. A. Asenjo, J. M. Pinto, Optimal process synthesis for the production of multiple recombinant proteins, Biotechnology Progress 20 (4) (2004) 1032-1043.

[30] R. J. Allgor, P. I. Barton, Mixed-integer dynamic optimization I: problem formulation, Computers \& Chemical Engineering $23(4-5)(1999)$ 567-584.

[31] A. A. Linninger, A. Chakraborty, Synthesis and optimization of waste treatment flowsheets, Computers \& Chemical Engineering 23 (10) (1999) 1415-1425.

[32] A. Chakraborty, A. A. Linninger, Plant-Wide Waste Management. 1. Synthesis and Multiobjective Design, Industrial \& Engineering Chemistry Research 41 (18) (2002) 4591-4604.

[33] L. L. Simon, N. Osterwalder, U. Fischer, K. Hungerbühler, Systematic Retrofit Method for Chemical Batch Processes Using Indicators, Heuristics, and Process Models, Industrial \& Engineering Chemistry Research 47 (1) (2008) 66-80.

[34] A. Carvalho, H. A. Matos, R. Gani, Design of batch operations: Systematic methodology for generation and analysis of sustainable alternatives, Computers \& Chemical Engineering 33 (12) (2009) 2075-2090.

[35] A. A. Bumann, S. Papadokonstantakis, U. Fischer, K. Hungerbühler, Investigating the use of path flow indicators as optimization drivers in batch process retrofitting, Computers \& Chemical Engineering 35 (12) (2011) 2767-2785.

[36] I. Halim, A. Carvalho, R. Srinivasan, H. A. Matos, R. Gani, A combined heuristic and indicator-based methodology for design of sustainable chemical process plants, Computers \& Chemical Engineering 35 (8) (2011) 1343-1358.

[37] A. Banimostafa, S. Papadokonstantakis, K. Hungerbühler, Retrofit design of a pharmaceutical batch process improving green process chemistry \& engineering principles, in: I. A. Karimi, R. Srinivasan (Eds.), 11th International Symposium on Process Systems Engineering, vol. 31 of Computer Aided Chemical Engineering, Elsevier, 1120-1124, 2012.

[38] T. Bhatia, L. T. Biegler, Dynamic optimization in the design and scheduling of multiproduct batch plants, Industrial \& Engineering Chemistry Research 35 (7) (1996) 2234-2246.

[39] Y. Nie, L. T. Biegler, J. M. Wassick, Integrated scheduling and dynamic optimization of batch processes using state equipment networks, AIChE Journal 58 (11) (2012) 3416-3432.

[40] K. Frankl, J. Beenken, W. Marquardt, Integrated Scheduling and Control of Continuous-Time Blending Processes, in: I. A. Karimi, R. Srinivasan (Eds.), 11th International Symposium on Process Systems Engineering, vol. 31 of Computer Aided Chemical Engineering, Elsevier, 1090-1094, 2012. 
[41] E. Capón-García, G. Guillén-Gosálbez, A. Espuña, Integrating process dynamics within batch process scheduling via mixed-integer dynamic optimization, Chemical Engineering Science 102 (2013) 139-150.

[42] Y. Chu, F. You, Integrated scheduling and dynamic optimization of network batch processes, in: American Control Conference (ACC), 2014, ISSN 0743-1619, 5024-5029, doi:10.1109/ACC.2014.6858593, 2014.

[43] K. C. Furman, Z. Jia, M. G. Ierapetritou, A Robust Event-Based Continuous Time Formulation for Tank Transfer Scheduling, Industrial \& Engineering Chemistry Research 46 (26) (2007) 9126-9136.

[44] S. Ferrer-Nadal, E. Capón-García, C. A. Mendez, L. Puigjaner, Material Transfer Operations in Batch Scheduling. A Critical Modeling Issue, Industrial \& Engineering Chemistry Research 47 (20) (2008) 7721-7732.

[45] E. Smith, C. Pantelides, Design of reaction/separation networks using detailed models, Computers \& Chemical Engineering 19, Supplement 1 (0) (1995) 83-88.

[46] I. E. Grossmann, G. Guillén-Gosálbez, Scope for the application of mathematical programming techniques in the synthesis and planning of sustainable processes, Computers \& Chemical Engineering 34 (9) (2010) 1365-1376.

[47] E. Kondili, C. Pantelides, R. Sargent, A General Algorithm for Short- Term Scheduling of Batch Operations - 1. MILP Formulation, Computers \& Chemical Engineering 17 (2) (1993) 211-227, ISSN 0098-1354.

[48] C. C. Pantelides, Unified frameworks for the optimal process planning and scheduling, in: Proceedings of the 2nd Conference on the Foundations of Computer Aided Operations, 253, 1994.

[49] Y. Nie, Integration of Scheduling and Dynamic Optimization: Computational Strategies and Industrial Applications, Ph.D. thesis, Carnegie Mellon University, 2014.

[50] P. I. Barton, C. C. Pantelides, Modeling of combined discrete/continuous processes, AIChE Journal 40 (6) (1994) $966-979$.

[51] G. Corsano, J. M. Montagna, O. A. Iribarren, P. A. Aguirre, Heuristic method for the optimal synthesis and design of batch plants considering mixed product campaigns, Industrial \& Engineering Chemistry Research 46 (9) (2007) 2769-2780.

[52] E. Balas, Disjunctive Programming and a Hierarchy of Relaxations for Discrete Optimization Problems, SIAM Journal on Algebraic and Discrete Methods 6 (3) (1985) 466-486.

[53] I. E. Grossmann, Mixed-integer programming approach for the synthesis of integrated process flowsheets, Computers \& Chemical Engineering 9 (5) (1985) 463-482.

[54] M. Duran, I. E. Grossmann, An outer-approximation algorithm for a class of mixed-integer nonlinear programs, Mathematical Programming 36 (1986) 307-339.

[55] R. Raman, I. E. Grossmann, Relation between MILP modelling and logical inference for chemical process synthesis, Computers \& Chemical Engineering 15 (2) (1991) 73-84.

[56] C. A. Floudas, I. E. Grossmann, Algorithmic approaches to Process Synthesis: Logic and Global Optimization, in: L. T. Biegler, M. F. Doherty (Eds.), Foundations of Computer-Aided Process Design, 198-221, 1994.

[57] A. Bemporad, M. Morari, Control of systems integrating logic, dynamics, and constraints, Automatica 35 (3) (1999) $407-427$.

[58] V. Bansal, J. D. Perkins, E. N. Pistikopoulos, A Case Study in Simultaneous Design and Control Using Rigorous, MixedInteger Dynamic Optimization Models, Industrial \& Engineering Chemistry Research 41 (4) (2002) 760-778.

[59] A. Prata, J. Oldenburg, A. Kroll, W. Marquardt, Integrated scheduling and dynamic optimization of grade transitions for a continuous polymerization reactor, Computers \& Chemical Engineering 32 (3) (2008) 463-476.

[60] G. Corsano, P. A. Aguirre, O. A. Iribarren, J. M. Montagna, Batch Fermentation Networks Model for Optimal Synthesis, Design, and Operation, Industrial \& Engineering Chemistry Research 43 (15) (2004) 4211-4219.

[61] O. Stein, J. Oldenburg, W. Marquardt, Continuous reformulations of discrete-continuous optimization problems, Computers \& Chemical Engineering 28 (10) (2004) 1951-1966.

[62] C. Neuman, A. Sen, A suboptimal control algorithm for constrained problems using cubic splines, Automatica 9 (5) (1973) $601-613$. 
[63] T. Tsang, D. Himmelblau, T. Edgar, Optimal control via collocation and nonlinear-programming, International Journal of Control 21 (5) (1975) 763-768.

[64] M. Moreno-Benito, C. Dombayci, A. Espuña, L. Puigjaner, Integrated Process and Plant Design Optimisation of Industrial Scale Batch Systems: Addressing the Inherent Dynamics through Stochastic and Hybrid Approaches, Chemical Engineering Transactions 45 (2015) 1789-1794.

[65] M. Türkay, I. E. Grossmann, Tight mixed-integer optimization models for the solution of linear and nonlinear systems of disjunctive equations, Computers \& Chemical Engineering 22 (9) (1998) 1229-1239.

[66] W. F. Clocksin, C. S. Mellish, Programming in Prolog, Springer-Verlag, New York, 1981.

[67] J. Cuthrell, L. Biegler, Simultaneous optimization and solution methods for batch reactor control profiles, Computers \& Chemical Engineering 13 (1-2) (1989) 49-62.

[68] I. E. Grossmann, Review of nonlinear mixed-integer and disjunctive programming techniques, Optimization \& Engineering 3 (2002) 227-252.

[69] R. E. Rosenthal, GAMS: A User's Guide, Scientific Press, 2012.

[70] K. Denbigh, Optimum temperature sequences in reactors, Chemical Engineering Science 8 (1-2) (1958) $125-132$.

[71] R. Giudici, Polymerization Reaction Engineering: A Personal Overview of the State of Art, Latin American Applied Research 30 (2000) 351-356.

[72] P. Bajaj, K. Sen, S. H. Bahrami, Solution polymerization of acrylonitrile with vinyl acids in dimethylformamide, Journal of Applied Polymer Science 59 (10) (1996) 1539-1550.

[73] M. D. Gol'dfein, B. A. Zyubin, Kinetics and mechanism of the processes of preparing fibre-forming polymers based on acrylonitrile - Review, Polymer Science U.S.S.R. 32 (11) (1990) 2145-2166.

[74] E. Capón-García, A. D. Bojarski, A. Espuña, L. Puigjaner, Multiobjective optimization of multiproduct batch plants scheduling under environmental and economic concerns, AIChE Journal 57 (10) (2011) 2766-2782. 


\section{List of Figures}

1 SEN superstructure of the motivating example $1 \ldots \ldots \ldots \ldots \ldots$

2 Two-level superstructure distribution of the motivating example 1. . . . . . . . . . . . . 40

3 Multistage and single-stage models of the motivating example 1 to represent batch and semicontinuous plant elements respectively. . . . . . . . . . . . . . . . . . . 41

4 Synchronization of batch stages and flow rates at Levels 0 and 1 for configurations $\alpha, \beta, \pi$, and $\sigma$ of the motivating example 1 , with $\left|K_{j}\right|=3, \forall j \in\left\{U_{1}, U_{2}\right\} \ldots \ldots \ldots \ldots \ldots$

$5 \quad$ Input/output variables and stages of batch unit $j \in U$ models, considering $m^{i n} \in M_{j}^{i n}$ input

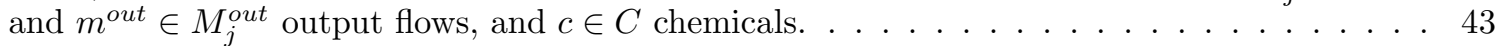

6 Petri net representing active and bypass stages $k \in K_{j}$ in the model of batch unit $j \in U$ at Level 1. Circles symbolize stages and bars represent stage-to-stage transitions. . . . . . . . . 44

7 SEN superstructure of the motivating example $2 \ldots \ldots \ldots \ldots \ldots$. . . . . . . . . 45

8 Petri net representing active stages $l \in L^{a}=\left\{1, \ldots,\left|L^{a}\right|\right\}$ and bypass stages $l \in L \backslash L^{a}=$ $\left\{\left|L^{a}\right|+1, \ldots, L^{\max }\right\}$ for semi-continuous elements at Level 0 . Grey elements represent feasible

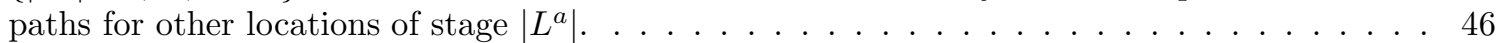

9 Optimal solution in the Denbigh example: operating mode and synchronization of unit pro-

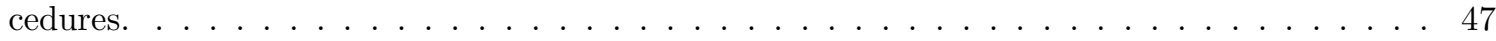

10 Control and process variable profiles in the fixed and optimal recipe i in the Denbigh example. 48

11 Temperature profiles in batch units in the Denbigh example for the fixed and optimal recipes in cases: (i) raw material cost minimization, (ii) profit maximization, (iii) profitability maximization, and (iv) profit maximization with capacity expansion. . . . . . . . . . . .

12 Process stages in acrylic fiber production system: general processing scheme (solid lines) and potential processing alternatives (dashed lines) . . . . . . . . . . . . . . .

13 Superstructure of the acrylic fiber example, composed of: solution and suspension polymerization reactors $R_{11}$ and $R_{12}$, evaporator $E_{2}$, washing and filtering units $F_{3}, F_{5}, F_{71}$ and $F_{72}$, repulping unit $R_{4}$, spinneret $S_{6}$, distillation columns $C_{81}$ and $C_{82}$, and buffer tanks $T_{2}, T_{3}, T_{4}$, $T_{81}$ and $T_{84}$. Equipment elements in black represent the addressed primary polymerization process for bulk polymer production. Equipment elements in grey represent the secondary part of the process for spun production. . . . . . . . . . . . . . .

14 Total cost for each subsystem and the integrated problem with and without recirculation and avoiding separation stage (i.e. $R_{2,1}=$ true, $R_{2,1}=$ false, $Z_{2}=$ false) in the acrylic fiber example. In black, integrated solution. . . . . . . . . . . . . . . . . 52

15 Optimal structure and logical variables in the acrylic fiber example. . . . . . . . . . . . 53

16 Control and process variable profiles of the polymerization reactor $R_{11}$ for the optimal solution in the acrylic fiber example: (1) flow rates / dosage and temperature and (2) molar

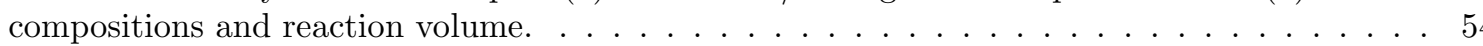

17 Control and process variable profiles of the polymerization reactor $R_{12}$ and the separator $E_{2}$ for the optimal solution with reduced amortization costs in the acrylic fiber example: (a1) flow rates / dosage and temperature, (b1) vapor flow, and (a2-b2) molar compositions and

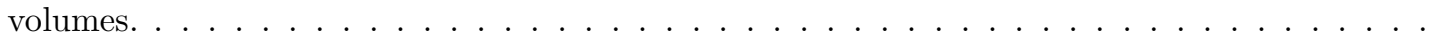




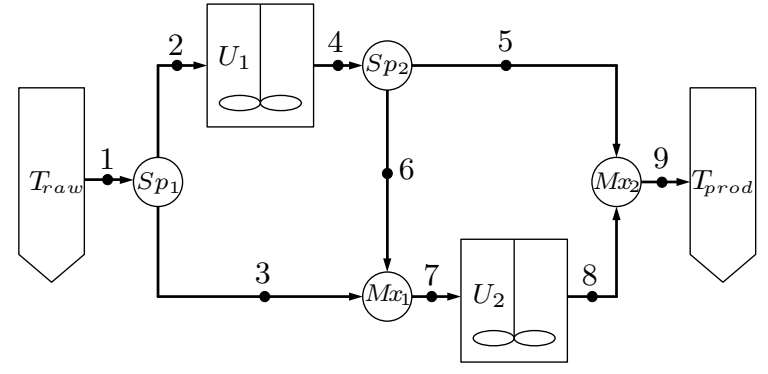

Figure 1 


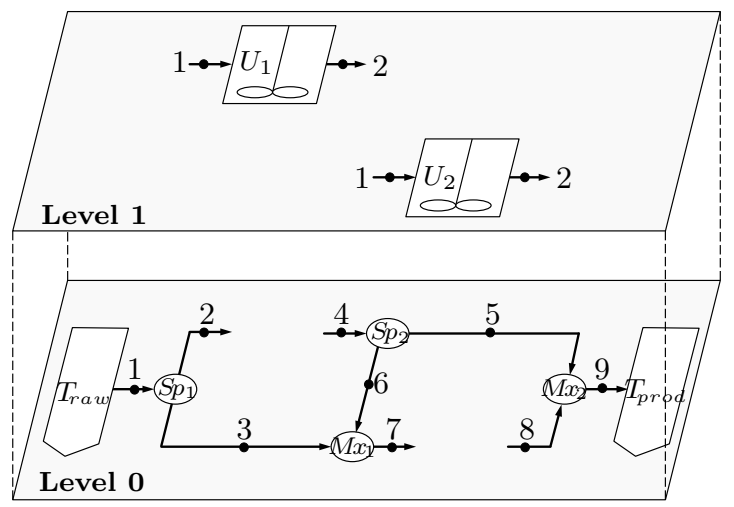

Figure 2 


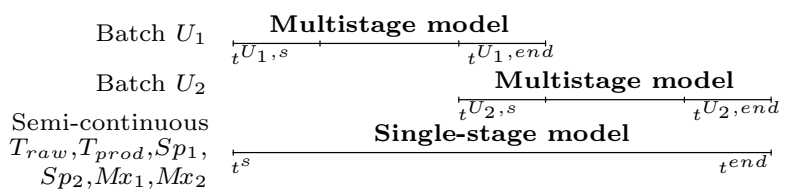

(a) Coexistence of models

\begin{tabular}{|c|c|c|c|c|}
\hline \multirow{2}{*}{ Batch $U_{1}$} & \multicolumn{2}{|c|}{ Multistage model } & & \\
\hline & \multirow{3}{*}{$t^{U_{1, s}}$} & \multirow{2}{*}{\multicolumn{3}{|c|}{$\begin{array}{r}\sigma_{1}, e n d \\
\text { Multistage model }\end{array}$}} \\
\hline Batch $U_{2}$ & & & & \\
\hline Semi-continuous & & Multistage & model & $t^{U_{2}, e n d}$ \\
\hline $\begin{array}{r}I_{\text {raw }}, I_{\text {prod }}, S p_{1}, \\
S p_{2}, M x_{1}, M x_{2}\end{array}$ & $t^{s}$ & & & \\
\hline
\end{tabular}

(b) Single-stage to multistage models at Level 0

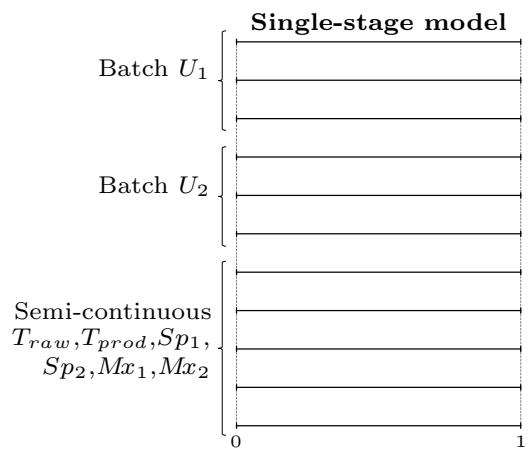

(c) Normalization of mathematical stages

Figure 3 


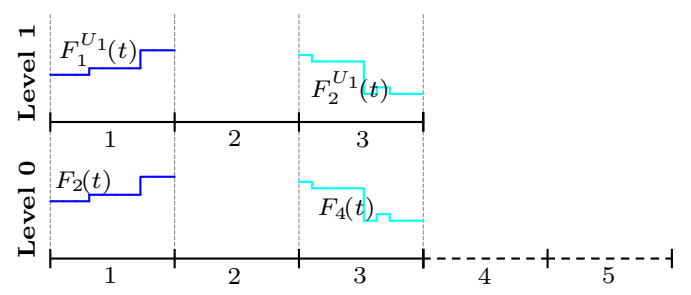

(a) $\alpha$ : single $U_{1}$

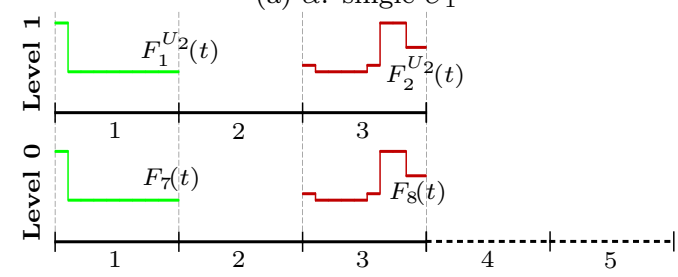

(b) $\beta$ : single $U_{2}$

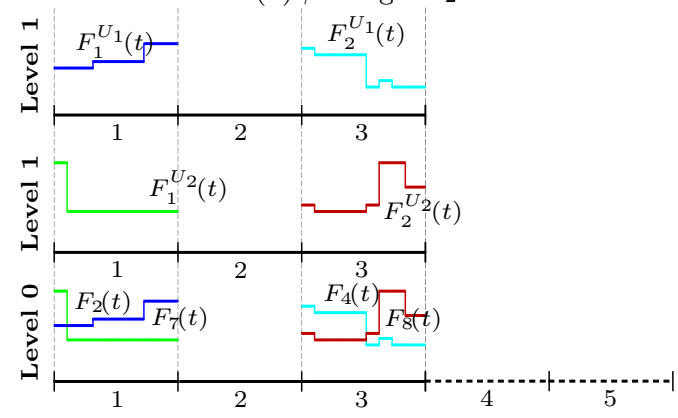

(c) $\pi$ : parallel in-phase $U_{1} \& U_{2}$

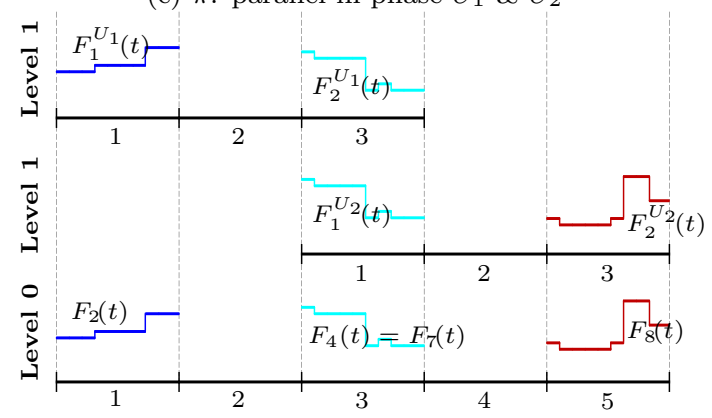

(d) $\sigma$ : series $U_{1}$ followed by $U_{2}$

Figure 4 


\begin{tabular}{|c|c|c|}
\hline $\begin{array}{c}D A E \text { system } \\
\forall k \in I_{j} \backslash O_{j}\end{array}$ & $\begin{array}{l}D A E \text { system } \\
\forall k \in K_{j} \backslash I_{j} \backslash O_{j}\end{array}$ & $\begin{array}{c}D A E \text { system } \\
\forall k \in O_{j} \backslash I_{j}\end{array}$ \\
\hline$\nabla_{x_{c, m^{j}}^{m^{\text {out }}, k}, k}$ & $0 F_{m^{j}}^{j}{ }^{\text {out }, k}(t)$ & $\downarrow \begin{array}{l}F_{m^{\text {out }}, k}^{j}(t) \\
x_{c, \text { mout }, k}^{j}(t)\end{array}$ \\
\hline
\end{tabular}

Figure 5 


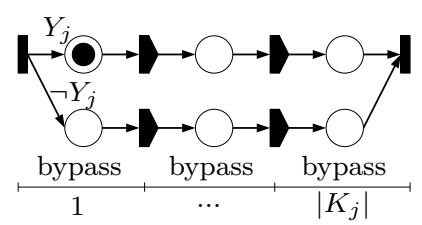

Figure 6 


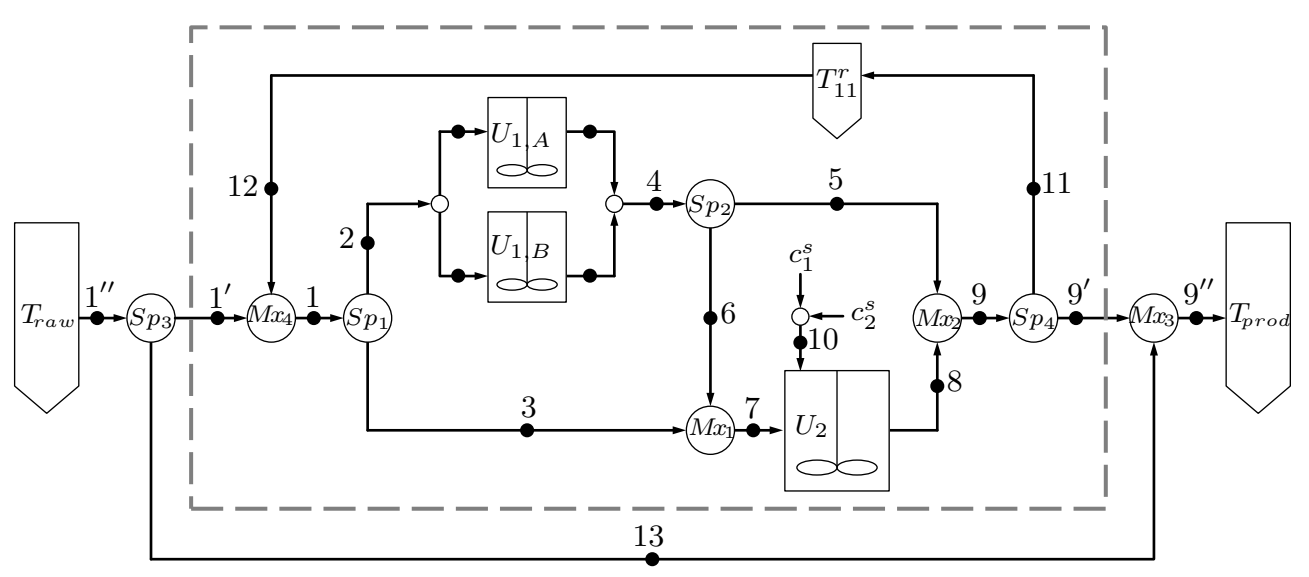

Figure 7 


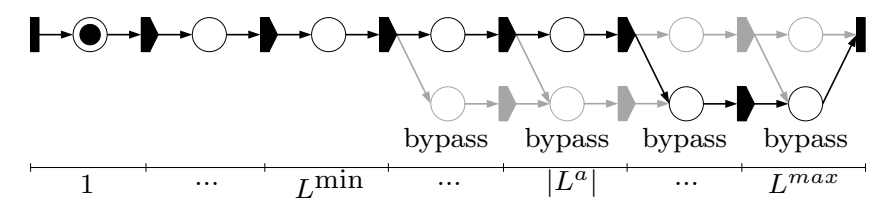

Figure 8 


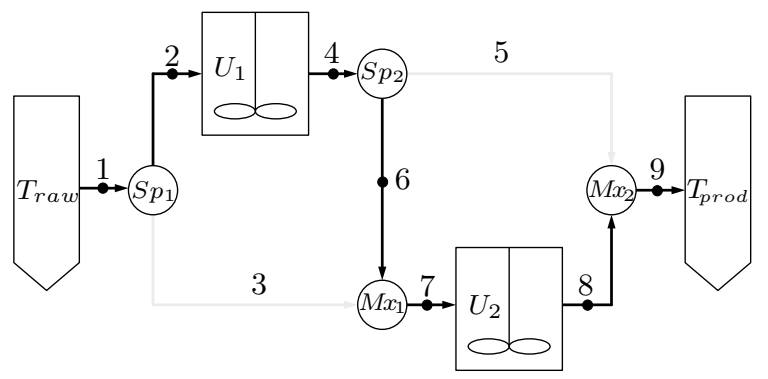

(a) Operating mode $\sigma$

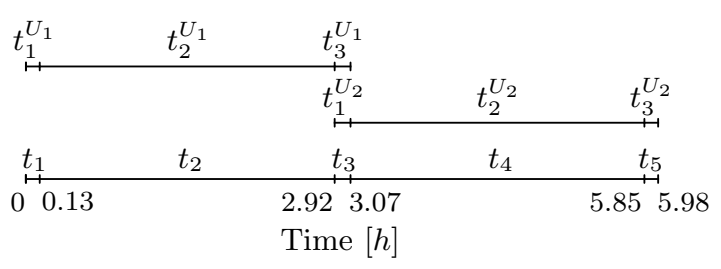

(b) Synchronization of unit procedures

Figure 9 
Fixed recipe
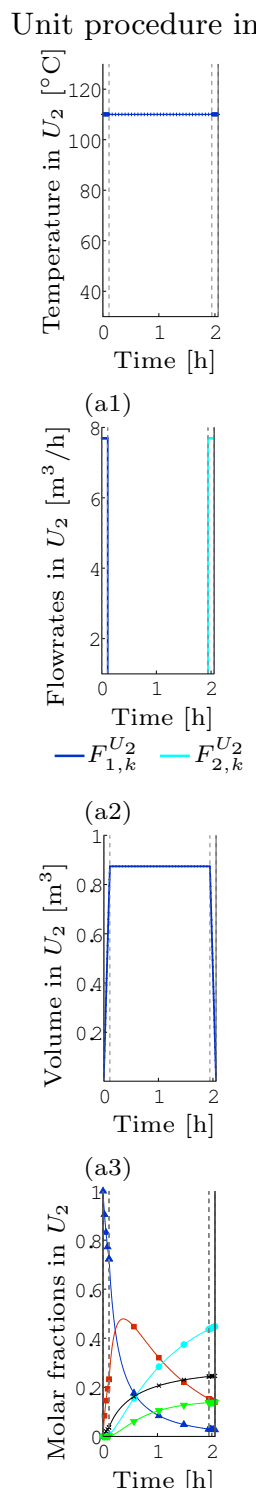

$\left\llcorner x_{\mathrm{A}, 2, k}^{U_{2}}-x_{\mathrm{R}, 2, k}^{U_{2}}\right.$

$-x_{\mathrm{S}, 2, k}^{U_{2}} * x_{\mathrm{T}, 2, k}^{U_{2}}$

$\nabla-x_{\mathrm{U}, 2, k}^{U_{2}}$

(a4)
Optimal recipe i
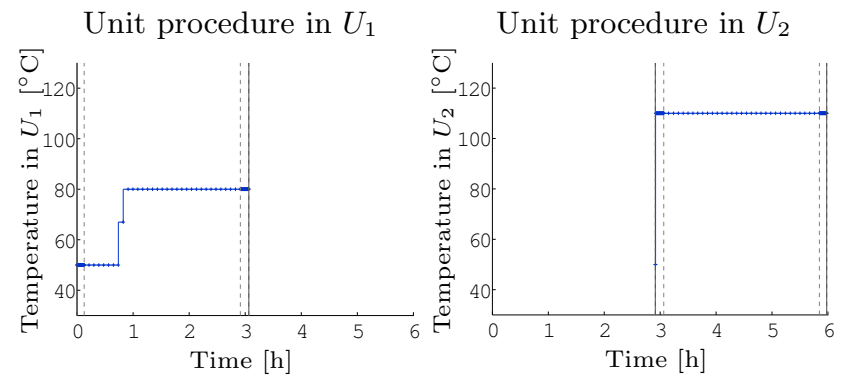

(b1)
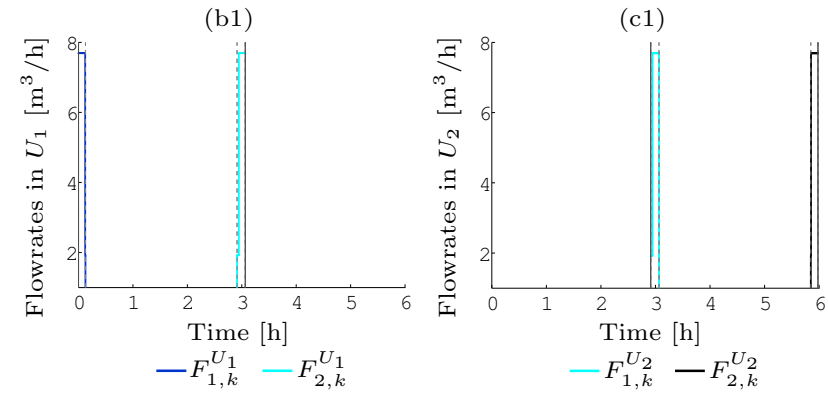

(b2)

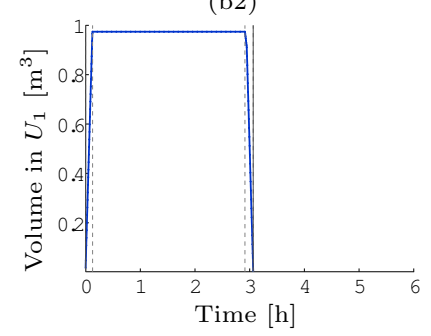

(c2)

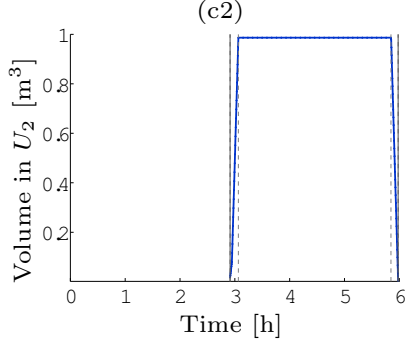

(b3)

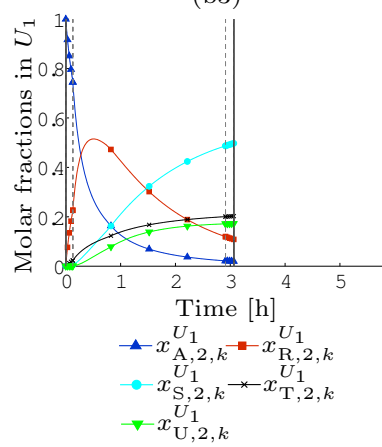

(b4)

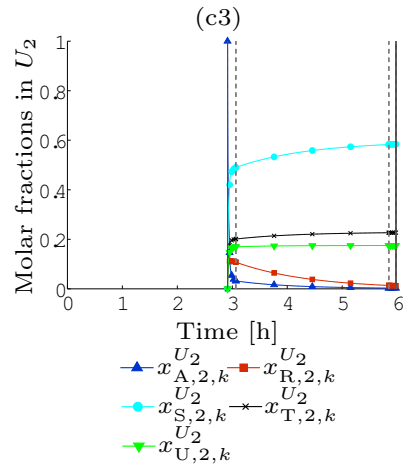

(c4)

Figure 10 

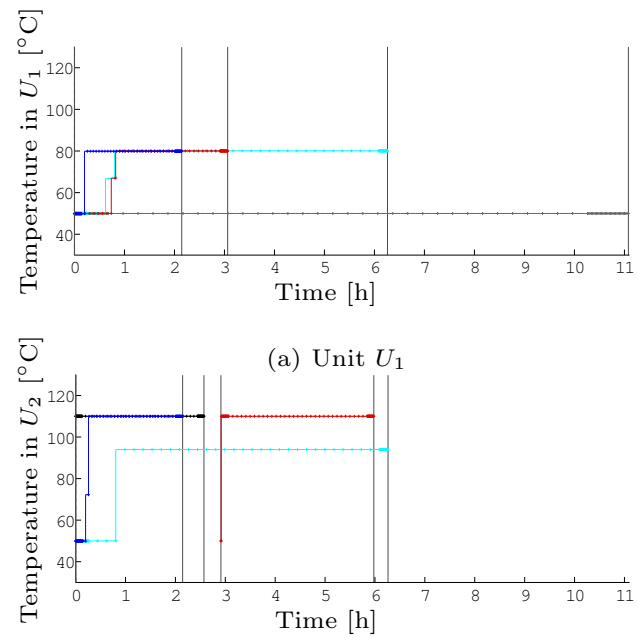

(b) Unit $U_{2}$

- Fixed recipe - Optimal recipe i - Optimal recipe ii - Optimal recipe iii — Optimal recipe iv

Figure 11 


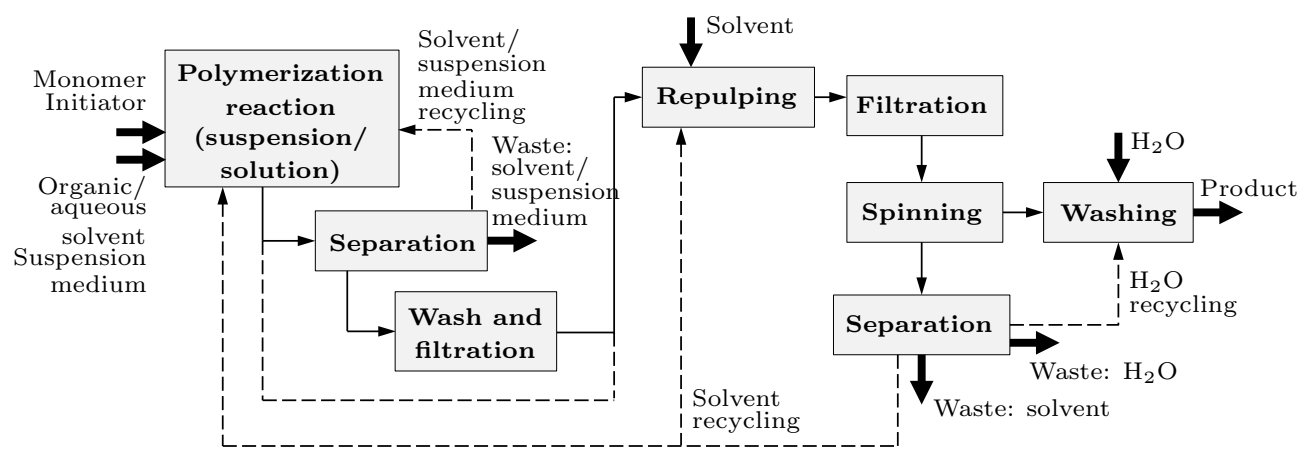

Figure 12 


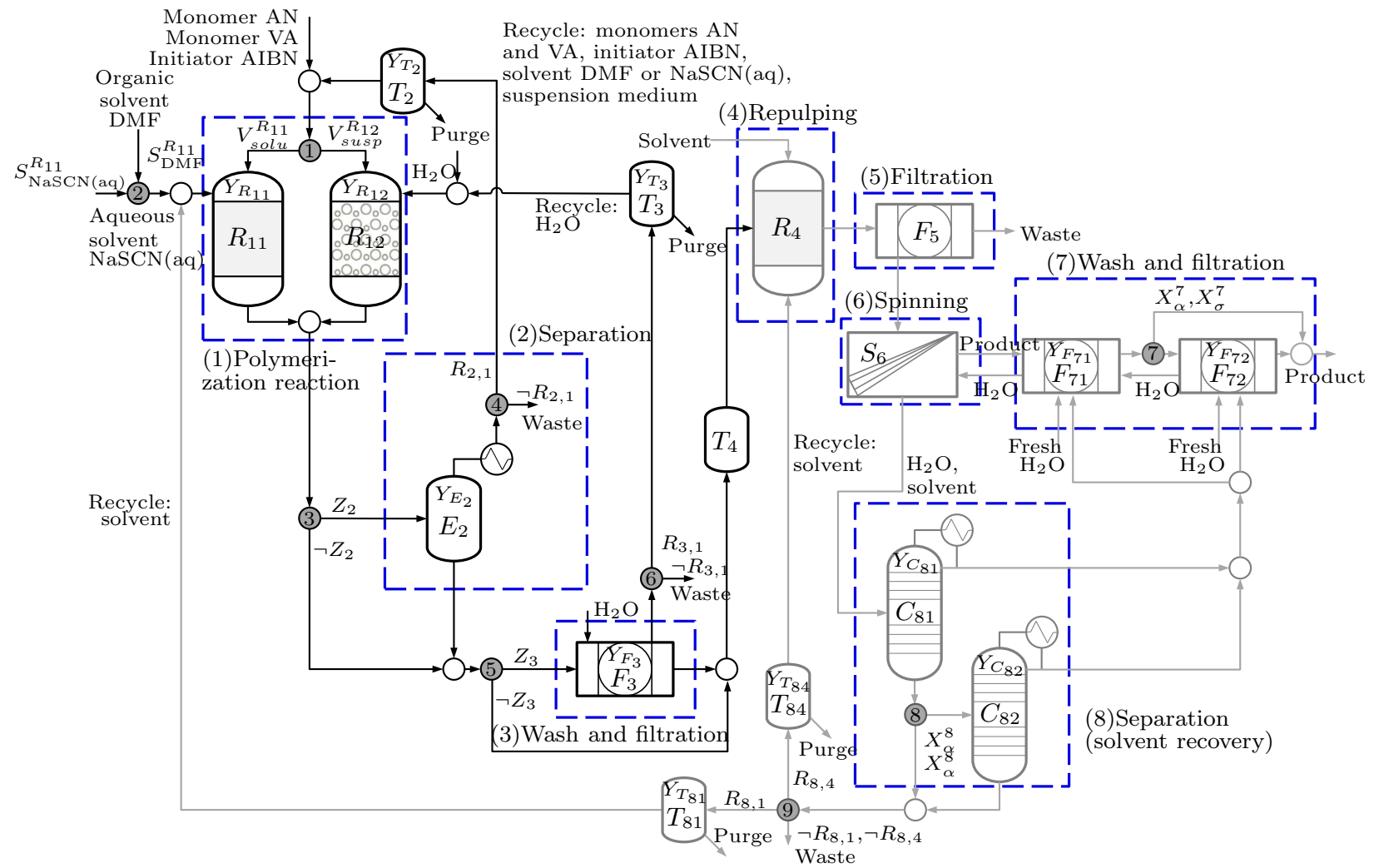

Figure 13 


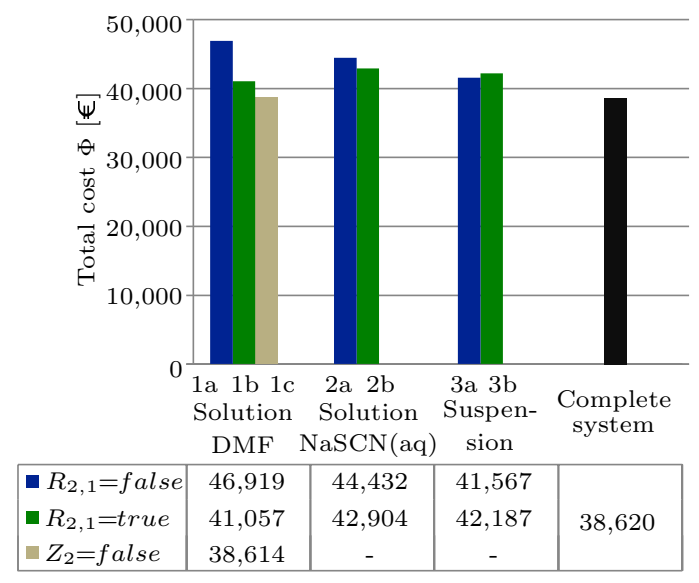

Figure 14 


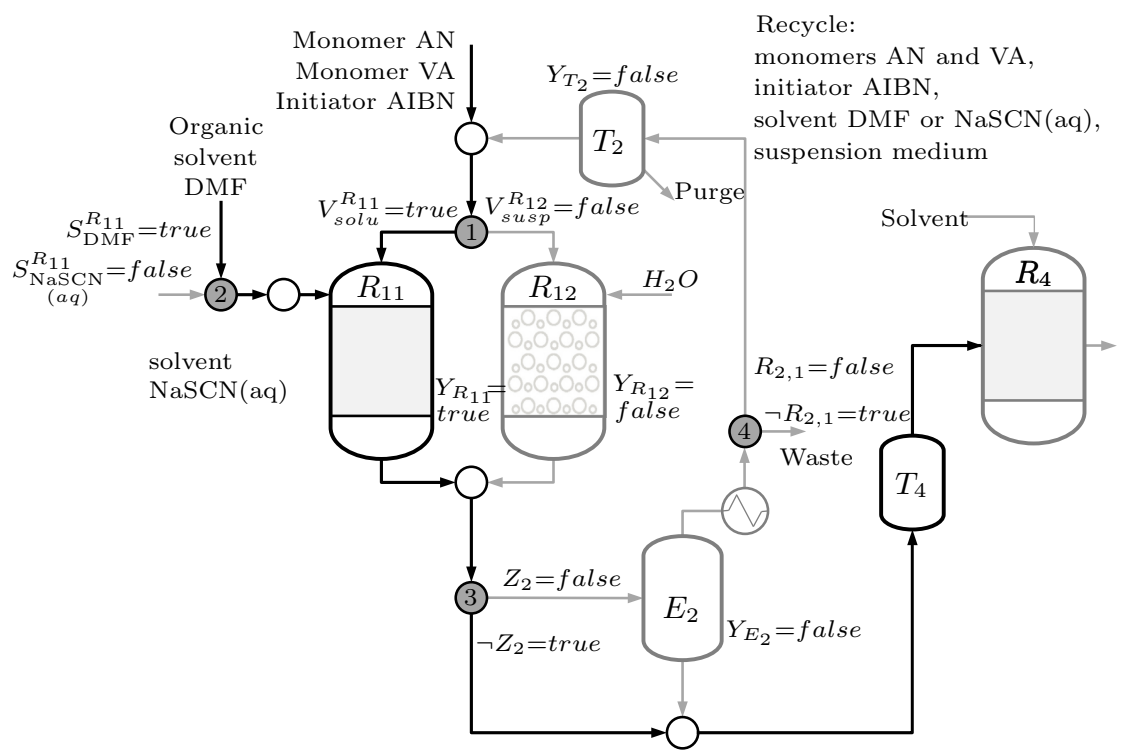

Figure 15 


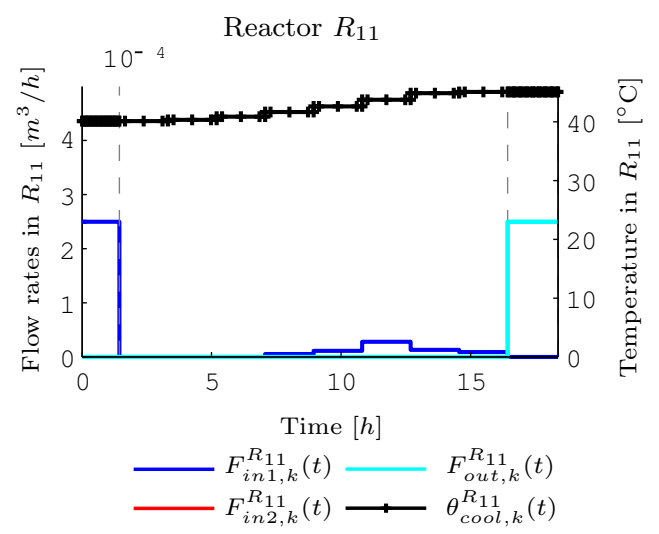

(1)

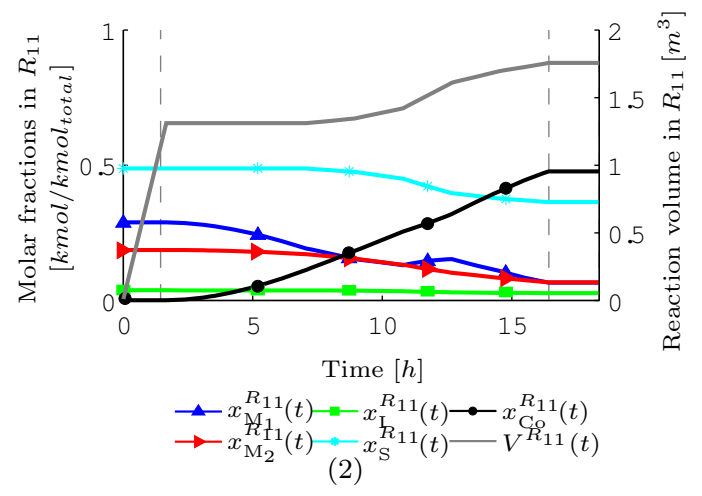

Figure 16 


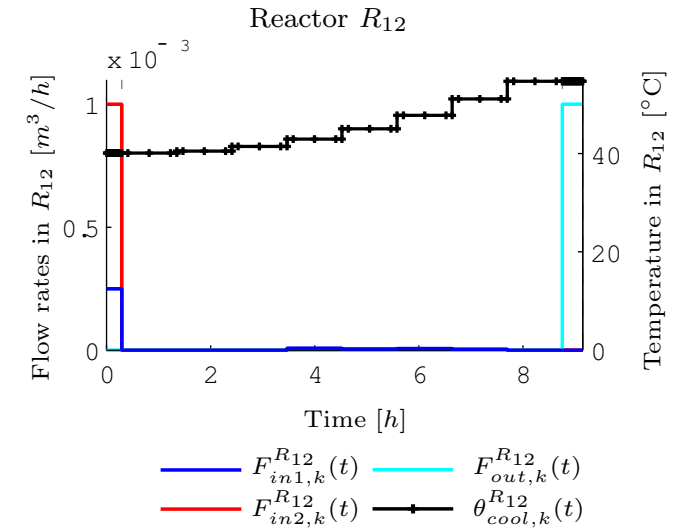

(a1)

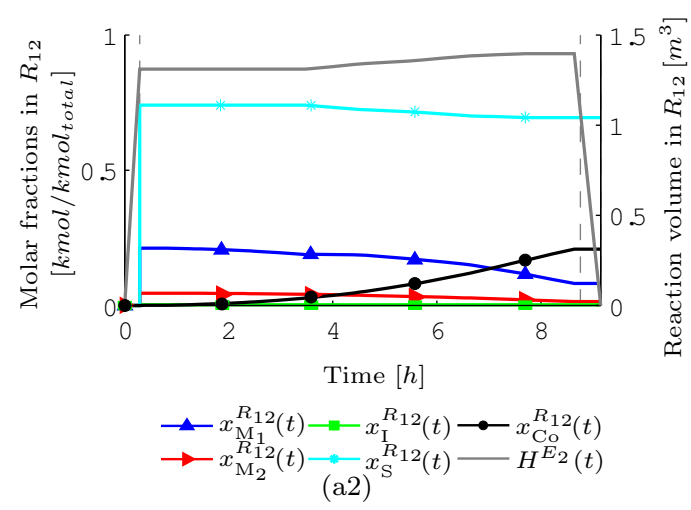

Evaporator $E_{2}$

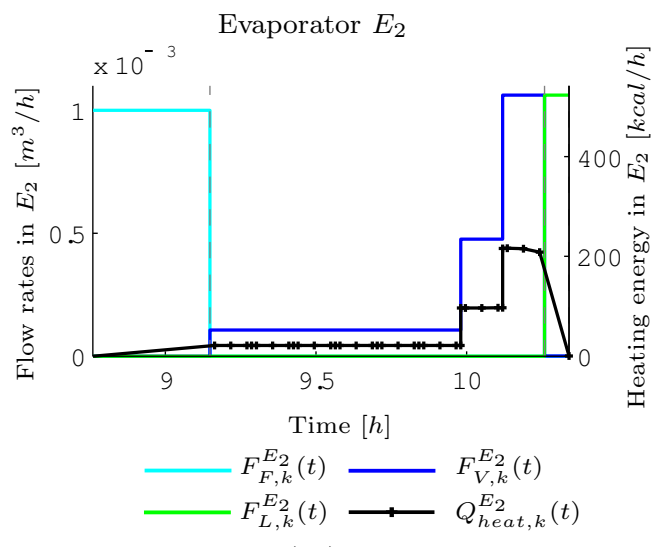

(b1)

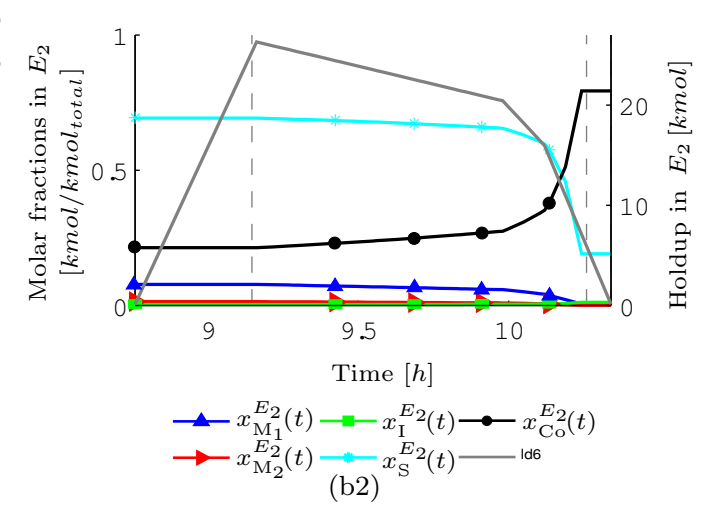

Figure 17 


\section{List of Tables}

1 Features of the MINLP models implemented in GAMS in the Denbigh example, obtained using 32 finite elements and 3 collocation points in the discretization step: IFS solutions with fixed configurations $X_{\psi}^{1}=$ true, $\psi \in\{\alpha, \beta, \pi, \sigma\}$ and complete MINLP problem initialized with IFS $4 . \ldots \ldots \ldots \ldots \ldots \ldots \ldots \ldots \ldots$

2 KPIs and individual economic weights in the Denbigh example: fixed recipe, dynamically optimal recipe in $U_{2}$, structurally optimal recipe with constant control profiles, and simultaneous optimal recipes for all DOF in the problem statement in cases: (i) raw material cost minimization, (ii) profit maximization, (iii) profitability maximization, and (iv) profit maximization with capacity expansion. Items in bold correspond to the objective function. . 58

3 Features of the MINLP models implemented in GAMS in the acrylic fiber example, obtained using 8 finite elements and 3 collocation pints in the discretization step: IFS solutions with problem subsystems $1,3,5$, and 7 and complete MINLP problem initialized with IFS $3 . \ldots 59$

4 KPIs and individual economic weights in the acrylic fiber example considering: subsystem alternatives 1 to 7 and the complete MINLP problem. Items in bold correspond to the total cost minimization objective. . . . . . . . . . . . . . . . . . 


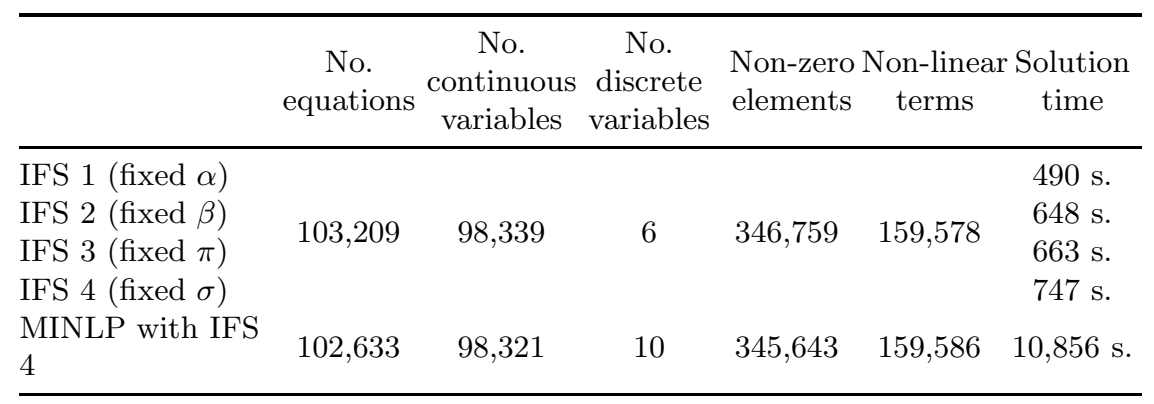

Table 1 
KPI

Fixed Dynamical- Structural- Optimal Optimal Optimal Optimal recipe ly optimal ly optimal recipe i recipe ii recipe iii recipe iv

\begin{tabular}{lccccccc}
\hline Equipment configuration & $\beta$ & $\beta$ & $\sigma$ & $\sigma$ & $\pi$ & $\pi$ & $\alpha$ \\
No. Batches & 70 & 54 & 48 & 47 & 23 & 31 & 13 \\
Batch size $[k g /$ batch] & 300 & 389 & 438 & 447 & 913 & 677 & 1,615 \\
Total processing time $[h]$ & 144.03 & 144.03 & 144.03 & 144.03 & 144.03 & 66.49 & 144.03 \\
Batch processing time $[h /$ batch] & 2.06 & 2.67 & 5.88 & 5.98 & 6.26 & 2.14 & 11.08 \\
Batch cycle time $[h /$ batch] & 2.06 & 2.67 & 3.00 & 3.06 & 6.26 & 2.14 & 11.08 \\
Total Profit $[€]$ & 4,764 & 5,332 & 5,037 & 5,097 & $\mathbf{5 , 8 4 1}$ & 4,861 & $\mathbf{5 , 9 4 8}$ \\
$\quad$ Total revenue & 9,046 & 9,046 & 9,046 & 9,046 & 9,046 & 9,046 & 9,046 \\
Raw material cost & 2,250 & $\mathbf{1 , 9 8 9}$ & $\mathbf{1 , 7 1 5}$ & $\mathbf{1 , 6 8 7}$ & 1,696 & 2,286 & 1,692 \\
Processing cost in $U$ & 967 & 899 & 824 & 822 & 789 & 955 & 729 \\
Occupation cost in $U$ & 1,065 & 825 & 1,470 & 1,440 & 720 & 944 & 535 \\
Amortization in $U$ & 0 & 0 & 0 & 0 & 0 & 0 & 142 \\
Penalty & 0 & $\mathbf{0}$ & $\mathbf{0}$ & $\mathbf{0}$ & 0 & 0 & 0 \\
Profit per batch $[€ /$ batch] & 68 & 113 & 107 & 108 & 254 & 157 & 458 \\
Profitability $[€ / h]$ & 33 & 37 & 35 & 35 & 41 & $\mathbf{7 3}$ & 41 \\
Selectivity of S $[$ kmol $\mathrm{S}$ & 0.448 & 0.507 & 0.588 & 0.597 & 0.595 & 0.441 & 0.596 \\
Total energy consumption $[k W h]$ & 38,692 & 35,973 & 32,974 & 32,879 & 31,558 & 38,211 & 29,159 \\
\hline
\end{tabular}

Table 2 


\begin{tabular}{|c|c|c|c|c|c|c|}
\hline & $\begin{array}{c}\text { No. } \\
\text { equations }\end{array}$ & $\begin{array}{c}\text { No. } \\
\text { continuous } \\
\text { variables }\end{array}$ & $\begin{array}{c}\text { No. discrete } \\
\text { variables }\end{array}$ & $\begin{array}{l}\text { Non-zero } \\
\text { elements }\end{array}$ & $\begin{array}{c}\text { Non-linear } \\
\text { terms }\end{array}$ & Solution time \\
\hline IFS 1 (subsystem 1) & & & & & & $85 \mathrm{~s}$. \\
\hline IFS 3 (subsystem 3) & 16.860 & 8.285 & 4 & 66.608 & 15.996 & $95 \mathrm{~s}$. \\
\hline IFS 5 (subsystem 5) & 10,000 & 0,200 & 4 & 00,000 & 15,990 & $65 \mathrm{~s}$. \\
\hline IFS 7 (subsystem 7) & & & & & & $101 \mathrm{~s}$. \\
\hline MINLP with IFS 3 & 16,860 & 8,285 & 14 & 66,608 & 15,996 & $338 \mathrm{~s}$. \\
\hline
\end{tabular}

Table 3 


\begin{tabular}{|c|c|c|c|c|c|c|c|c|c|}
\hline & & \multicolumn{7}{|c|}{ Subsystems } & \multirow{2}{*}{$\begin{array}{l}\text { Inte- } \\
\text { grated }\end{array}$} \\
\hline & & 1 & 2 & 3 & 4 & 5 & 6 & 7 & \\
\hline \multicolumn{2}{|c|}{ Polymerization type } & \multicolumn{3}{|c|}{ Solution } & \multicolumn{2}{|c|}{ Solution } & \multicolumn{2}{|c|}{ Suspension } & Sol. \\
\hline \multicolumn{2}{|c|}{ Solvent type } & \multicolumn{3}{|c|}{ DMF } & \multicolumn{2}{|c|}{$\operatorname{NaSCN}(\mathrm{aq})$} & \multicolumn{2}{|c|}{-} & $\mathrm{DMF}$ \\
\hline \multicolumn{2}{|c|}{ Process structure $\left(Z_{2}, R_{2,1}\right)$} & $(1,0)$ & $(1,1)$ & $(0,0)$ & $(1,0)$ & $(1,1)$ & $(1,0)$ & $(1,1)$ & $(0,0)$ \\
\hline \multicolumn{2}{|c|}{ Equipment size $\left[\mathrm{m}^{3}\right]$} & 1.5 & 1.5 & 2 & 2.5 & 2 & 1.5 & 1.5 & 2 \\
\hline \multicolumn{2}{|c|}{ No. Batches } & 8 & 9 & 6 & 6 & 9 & 17 & 22 & 6 \\
\hline \multicolumn{2}{|c|}{ Batch size $[\mathrm{kg} /$ batch $]$} & 625 & 556 & 833 & 833 & 556 & 294 & 227 & 833 \\
\hline \multicolumn{2}{|c|}{ Total processing time $[h]$} & 119.9 & 134.9 & 89.9 & 89.9 & 86.2 & 144.0 & 144.0 & 89.9 \\
\hline \multicolumn{2}{|c|}{ Batch proc. Time $[h / b a t c h]$} & 18.0 & 18.2 & 18.3 & 19.9 & 11.9 & 11.6 & 8.4 & 18.4 \\
\hline \multicolumn{2}{|c|}{ Batch cycle time $[h /$ batch $]$} & 16.7 & 16.9 & 18.3 & 18.5 & 10.6 & 10.4 & 7.2 & 18.4 \\
\hline \multicolumn{2}{|c|}{ Total cost $[€]$} & 46,919 & 41,057 & 38,614 & 44,432 & 42,904 & 41,567 & 42,187 & 38,620 \\
\hline \multicolumn{2}{|c|}{$R_{11} \quad$ Amortization } & 12,234 & 12,234 & 14,083 & 15,716 & 14,083 & 12,234 & 12,234 & 14,083 \\
\hline \multicolumn{2}{|c|}{ or Water consumption cost } & 113 & 114 & 113 & 106 & 104 & 372 & 369 & 113 \\
\hline \multirow[t]{4}{*}{$R_{12}$} & Monomer $\mathrm{M}_{1}$ cost & 12,048 & 13,750 & 8,568 & 8,412 & 16,233 & 10,418 & 13,151 & 8,683 \\
\hline & Monomer $\mathrm{M}_{2}$ cost & 5,082 & 6,448 & 2,267 & 1,619 & 8,526 & 1,637 & 2,013 & 2,154 \\
\hline & Initator I cost & 3,355 & 2,876 & 7,449 & 2,258 & 1,114 & 1,653 & 2,145 & 7,296 \\
\hline & Solvent/suspension $\mathrm{S}$ cost & 1,192 & 692 & 2,824 & 732 & 236 & 99 & 129 & 2,981 \\
\hline \multirow[t]{3}{*}{$E_{2}$} & Amortization & 8,368 & 8,368 & - & 10,750 & 9,633 & 8,368 & 8,368 & - \\
\hline & Energy cost & 202 & 257 & - & 268 & 452 & 655 & 977 & - \\
\hline & Waste disposal cost & 1,016 & 1,130 & - & 1,262 & 1,530 & 1,980 & 2,571 & - \\
\hline \multicolumn{2}{|c|}{$\mathrm{T}_{2} \quad$ Amortization } & - & 3,309 & - & - & 3,309 & - & 3,309 & - \\
\hline \multicolumn{2}{|c|}{$T_{4} \quad$ Amortization } & 3,309 & 3,309 & 3,309 & 3,309 & 3,309 & 3,309 & 3,309 & 3,309 \\
\hline \multicolumn{2}{|c|}{ Cost per batch $[€ /$ batch $]$} & 5,865 & 4,562 & 6,436 & 7,405 & 4,767 & 2,445 & 1,918 & 6,437 \\
\hline \multicolumn{2}{|c|}{ Monomer conversion } & 0.44 & 0.37 & 0.75 & 0.83 & 0.29 & 0.71 & 0.56 & 0.75 \\
\hline
\end{tabular}

Table 4 
